\title{
JERK-FREE GENEVA WHEEL DRIVING
}

\author{
Dr. E. A. DIJKSMAN \\ Technological University, Eindhoven, The Netherlands
}

(Received 23 March 1966)

\begin{abstract}
A jerk-free motion of an internal Geneva wheel is obtained by using a special driving mechanism. For this mechanism a four-bar mechanism is chosen from which the coupler point drives the wheel. By special choice of the four-bar linkage the coupler curve can be made symmetrical and containing two Ball's points. For each number of stations the dimensions of the mechanism are tabled for optimal transmission angles and next to it also in those cases where the coupler point lies in one line with the two moving turningpoints.

Drawings of the intermittent mechanism are simplified in cases where the circling-point curve degenerates.
\end{abstract}

\section{INTRODUCTION}

NORMALLY a Geneva wheel is driven by a crank with a crankpin. At the beginning as well as at the end of the intermittent motion at least two grooves of the Geneva wheel are then tangential to the circle traced by the crankpin. At those positions the normal acceleration of the rotating crankpin will be transmitted to the wheel with an impact. Hence, the wheel has an infinitely great jerk $\nmid$ at such positions. This leads to an abrupt change in force on the wheel to the effect of undesirable vibrations in the mechanism.

With an internal Geneva wheel this drawback can be removed by using a four-bar linkage as a driving mechanism instead of a simple crank.

Such a four-bar linkage, indicated henceforth by $\left(A_{0} A B B_{0}\right)$, is a closed kinematic chain consisting of 4 links, one of them being the fixed link $\left(A_{0} B_{0}\right)$. The opposite link, the coupler $(A B)$, is the base of a coupler-triangle, the vertex of which, the coupler-point $K$, describes the coupler curve when the mechanism is moved (see Fig. 1).

If the driving pin is now situated in a coupler point it is possible to trace an inflection point at the moment of entering or leaving the Geneva wheel. The tangents to the coupler curve in the two symmetrically situated inflection points of the curve must then coincide with the corresponding grooves of the wheel.

To avoid undesirable turns in the coupler curve, it is still better to replace the inflection points by what is known as undulation points or Ball's points [4].

At a Ball's point the coupler curve has a four-point contact with its tangent, through which the coupler curve, at least for a great part, runs on one side of this tangent. As a consequence, the motion of the turning table can begin and end with a zero angular jerk.

$\dagger$ By jerk is understood the time-derivative of the acceleration. 


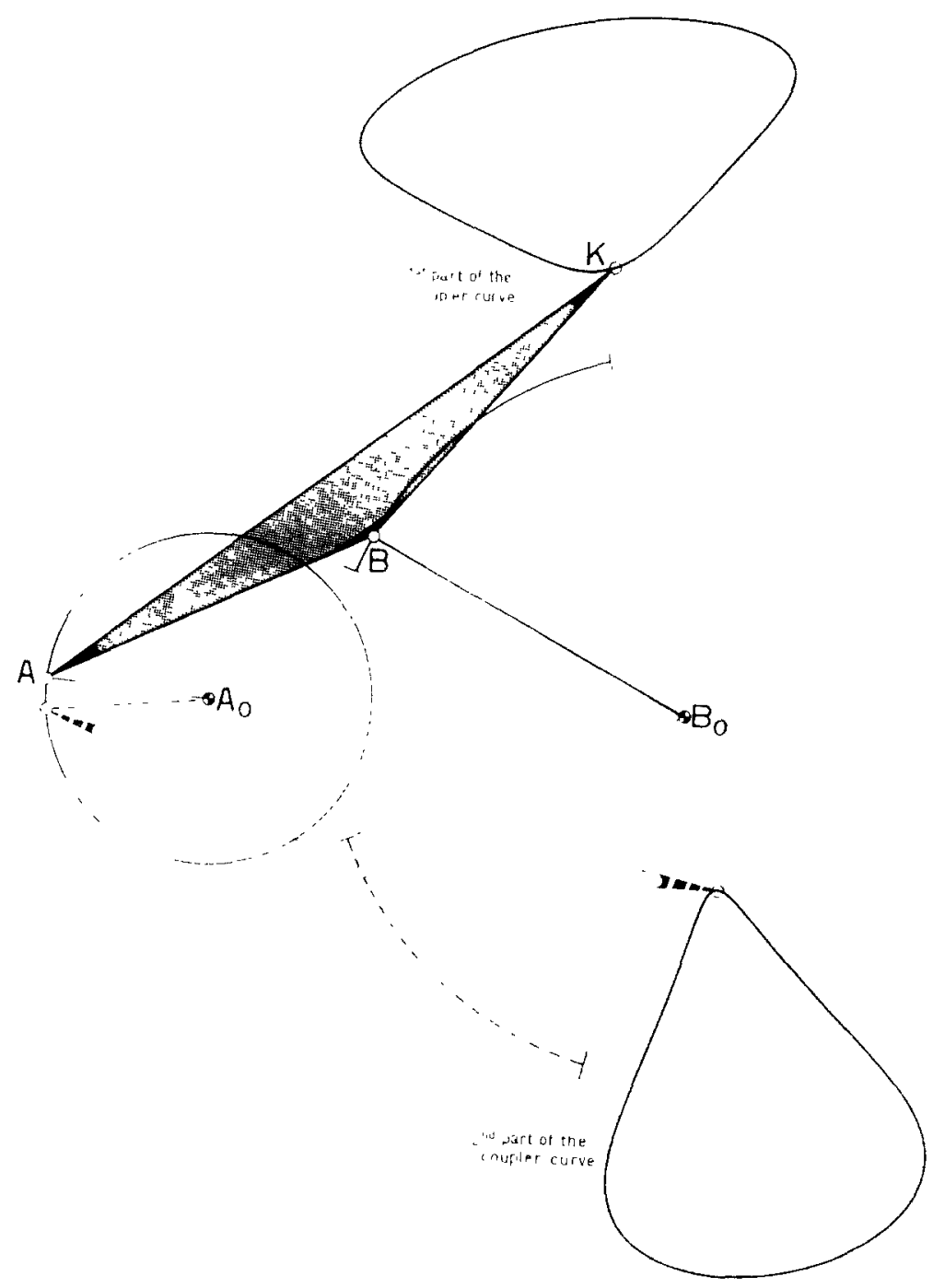

FIC. 1

If the acceleration period may be made equal to the deceleration period, it is convenient to make the coupler curve symmetrical. The problem is then to determine the dimensions of the four-bar linkage in such a way that the symmetrical coupler curve has a Ball's point which is not on the axis of symmetry and in which the tangent to the coupler curve makes a given angle $\tau$ with the axis of symmetry (see Fig. 2).

This problem has already been acknowledged by Hunt and his colleagues [1], who found a way to achieve a general solution of the problem. The present work deals with a further study on the subject, and is a condensation of the author's thesis [2] published in Dutch. The thesis contains a large number of graphs, from which the dimensions of the mechanism can be taken.

To drive the Geneva wheel, the input link has to make complete revolutions with respect to the fixed link. So only four-bar linkages complying with the condition of Grashof can be 


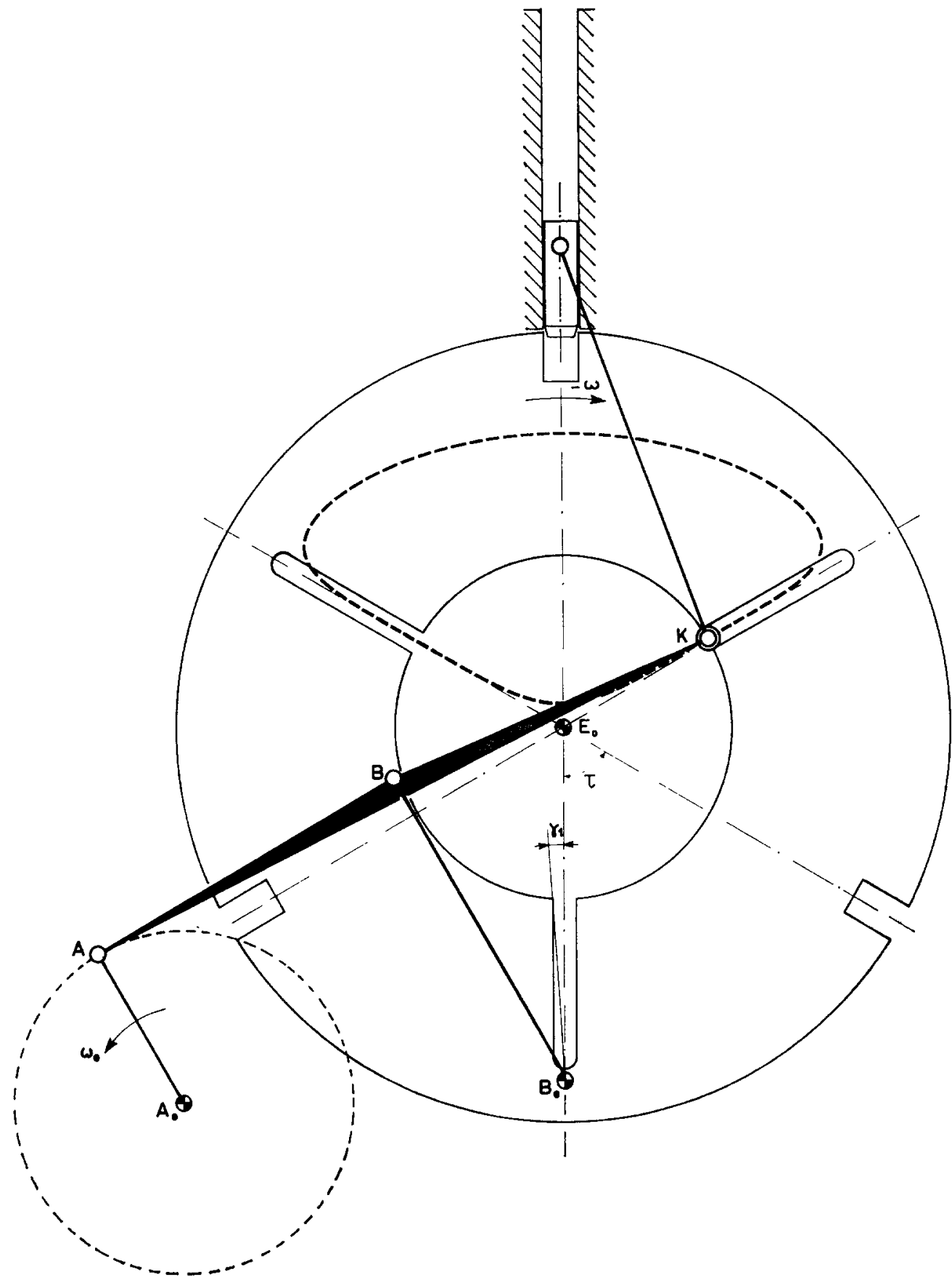

FIG. 2.

considered. According to whether $A_{0} B_{0}, A_{0} A$ or $A B$ is the smallest, we speak of a drag-link a crank-and-rocker or a double-rocker mechanism. The coupler of the last mechanism must be driven directly from the fixed link; if no gear wheels are used this is only possible by bringing the driving axle of the rotor in the moving pivot $A$. This is an extra complication, 
which however, can be avoided by using one of the cognate mechanisms corresponding to the proposition of Roberts instead of the double rocker mechanism. We then get a crankand-rocker mechanism with exactly the same coupler curve as the one we started from.

Up to the time of writing, no usable solutions of the problem have been found for the drag-link mechanism. We can, therefore, as far as the solutions are concerned, confine ourselves exclusively to the crank-and-rocker mechanisms.

We know that symmetrical coupler curves are generated if $\overrightarrow{B A}=\overrightarrow{B B}_{0}=\overrightarrow{B K}$. The axis of symmetry thereby goes through the fixed turning-point $B_{0}$ and makes an angle of $((\pi / 2)-$ $\Varangle K A B$ ) with the fixed link $A_{0} B_{0}$. It is also perpendicular to $A_{0} C_{0}$, a side of the fixed link triangle $A_{0} B_{0} C_{0}$, which is similar to the coupler-triangle $A B K$. Turning the coupler-triangle $180^{\circ}$ about its coupler causes the axis of symmetry also to turn $180^{\circ}$ about the fixed link. If the crankpoint arrives at the opposite point with respect to the fixed link, the coupler point coincides with its opposite position with respect to the axis of symmetry. Hence, the two positions of the crank-and-rocker mechanism with the crank-point $A$ in line with $A_{0} B_{0}$, have their corresponding coupler points at the intersections of the coupler curve with the axis of symmetry.

\section{KINEMATIC FUNDAMENTAL THEORY [3]}

The locus of those points of the moving coupler plane at which, at the moment of observation, the radius $\rho$ of curvature in the described curves has a stationary value, is called the circling-point curve $\dagger$ and is indicated by $k_{u}$. Thus, in accordance with this definition, $k_{u}$ is the locus of those coupler points for which $(\mathrm{d} \rho / \mathrm{d} t)=0$. Its equation in the polar co-ordinates $r$ and $\varphi$ reads:

$$
\frac{1}{r}=\frac{1}{l \sin \varphi}+\frac{1}{m \cos \varphi}
$$

The abscissa axis of the corresponding Cartesian co-ordinates coincides with the pole tangent $p$, and the ordinate with the pole normal $n$ (see Fig. 3). The positive sense of $y$ is taken in the direction from $P$ to the inflection pole $W$. The positive sense of $x$ changes into the positive sense of $y$ after rotating the abscissa axis counter-clockwise about $P$ through $\pi / 2$ radians.

If $X_{w}$ is the intersection of a path normal $P X$ with the inflection circle, the angle $\varphi$ is determined by the equations: $\varphi=\Varangle X_{w} P x$ where $0 \leqq \varphi<\pi$.

$r$ is the distance from the origin $P$ to the point of path $X$. If such a point is lying in the upper semi-plane, then $r$ is considered positive, in the other case negative.

The length $m$ is given by the equation:

$$
m=-\frac{3 \delta}{(\mathrm{d} \delta / \mathrm{d} s)}
$$

where $\delta$ is the diameter of the inflection circle and $s$ the length of arc along the fixed polode. $\delta$ is always positive.

The length $l$ is given by the equation

$$
\frac{3}{l}=\frac{1}{R}+\frac{1}{\delta}=\frac{2}{R}-\frac{1}{R_{0}}
$$

f Or cubic of stationary curvature. 


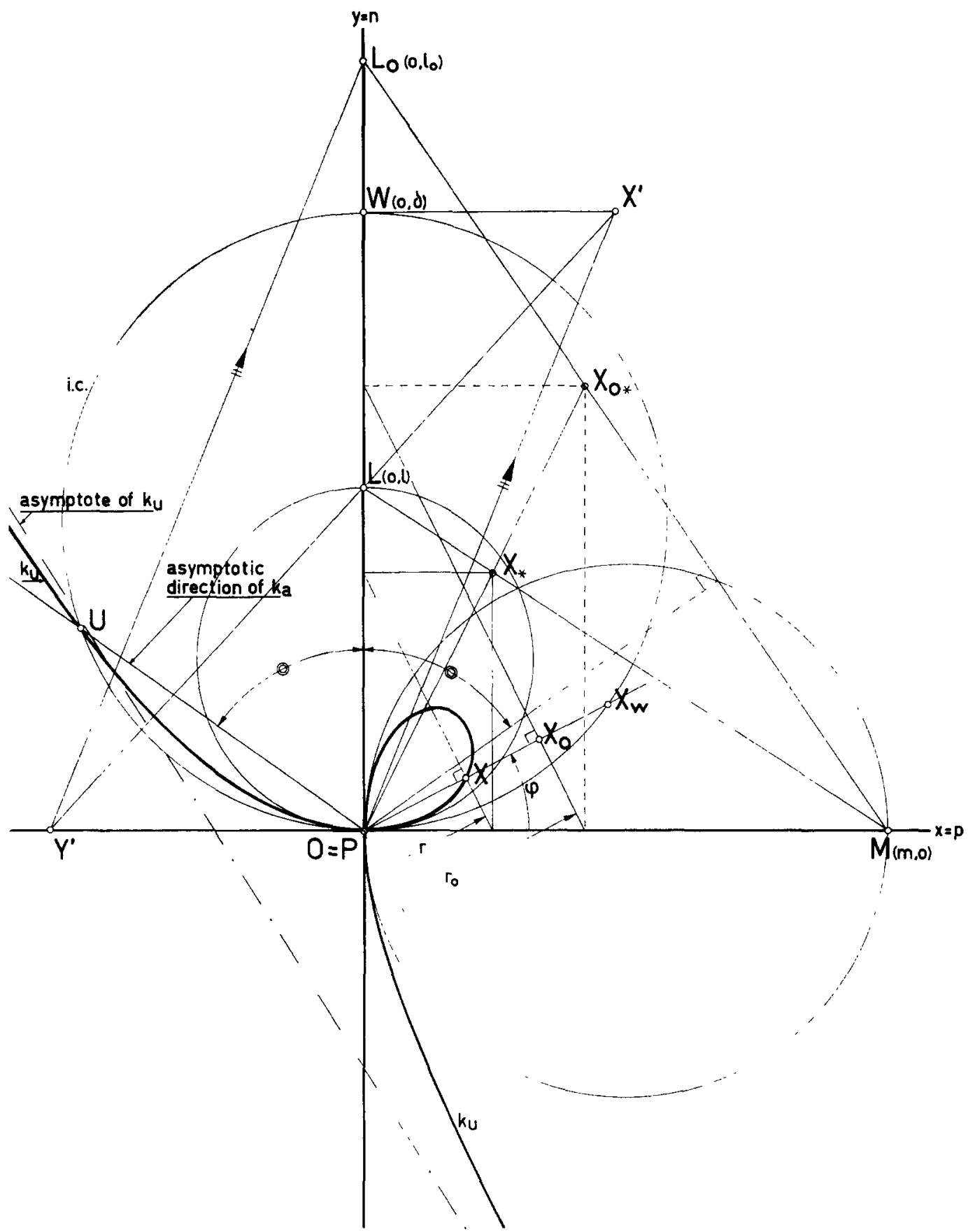

FIG. 3.

where $R$ and $R_{0}$ represent the ordinates of the centres of curvature of, respectively, the moving and the fixed polode in the pole. It is known that $k_{u}$ has a double-point in the origin $P$ with radii $\frac{1}{2} l$ and $\frac{1}{2} m$, the former coinciding with the pole normal, the latter with the pole tangent. 
In accordance with the definition, the turning-points $A$ and $B$ of a four-bar linkage $\left(A_{0} A B B_{0}\right)$ lie on the circling-point curve. Therefore, the circling-point curve can be considered to be the locus of possible points of choice $A$ and $B$ of the four-bar linkage.

The locus of those centres of curvature, of which the corresponding points of the path lie on the circling-point curve, is called the instantaneous centering-point curve and is indicated by $k_{a}$. The centering-point curve $k_{a}$ is the circling-point curve of the inverse motion.

In accordance with the definition the centres of pivot on frame, $A_{0}$ and $B_{0}$, of a four-bar linkage $\left(A_{0} A B B_{0}\right)$ lie on the centering point curve, so curve $k_{a}$ may also be seen as the locus of possible points of choice $A_{0}$ and $B_{0}$ of the four-bar linkage.

In the polar co-ordinates $r_{0}$ and $\varphi, k_{a}$ is given by the equation

$$
\frac{1}{r_{n}}=\frac{1}{l_{0} \sin \varphi}+\frac{1}{m \cos \varphi}
$$

where $l_{0}$ is defined by the equation

$$
\frac{3}{l_{0}}=\frac{2}{R_{0}}-\frac{1}{R}=\frac{1}{R_{0}}-\frac{1}{i}
$$

or by

$$
\frac{1}{l_{0}}=\frac{1}{l}-\frac{1}{\delta}
$$

\section{BALL'S POINT OR THE UNDULATION POINT $U$ [4]}

Ball's point is defined as that point of the path where the path tangent has a four-point contact with the path. This point lies on the inflection circle as well as on the circling-point curve. These curves generally intersect at $2 \times 3=6$ points, viz. the two isotropic points, the triple counting pole and a sixth point, Ball's point. If the pole is a triple intersection, a real Ball's point exists. In some special cases the inflection circle coincides with part of $k_{u}$ and then any point of the inflection circle may be considered to be a Ball's point.

Generally Ball's point is identified by intersecting the inflection circle with the line through the pole in the asymptotic direction of the centering-point curve. This direction is fixed by the direction coefficient $-m / l_{0}$ (see Fig. 3).

\section{DEGENERATIONS OF $k_{\|}$AND $k$}

Parts of this chapter which are printed in small type give rise to earlier derived driving mechanisms or to mechanisms unsuitable to our purpose.

\subsection{The general cycloidal position of the four-bar linkage}

If $\mathrm{d} \delta / \mathrm{d} s=0$ (or $m^{-1}=0$ ), it follows from (1) that either $\varphi=\frac{1}{2} \pi$, which is the equation of the pole normal, or that $r=l \sin \varphi$, which is the equation of a circle through $P$ with its centre on the pole normal.

In this case the $k_{u}$ curve is degenerated in the pole normal and a circle through $P(0,0)$ and $L(0, l)$ with its centre on the pole normal $n$. In the same way we find the $k_{a}$ curve to be 
degenerated in the pole normal and a circle through $P(0,0)$ and $L_{0}\left(0, l_{0}\right)$ with its centre on $n$.

The relation between the ordinates of the points $L(0, l), L_{0}\left(0, l_{0}\right)$ and the inflection pole $W(0, \delta)$ is expressed by

$$
\frac{1}{l}-\frac{1}{l_{0}}=\frac{1}{\delta}
$$

(see Fig. 9). Hence, the three circles are interdependent. This interdependence can be designed as follows:

(a) choose $X$ anywhere in the moving plane;

(b) connect $X$ with $P, L$ and $W$;

(c) draw $P Y$ parallel to $X W$ and find $Y$ on $X L$;

(d) draw $Y L$ parallel to $P X$ and find $L_{0}$ on $n$.

Proof. From

$$
\frac{\overline{P W}}{\overline{L_{0} P}}=\frac{\overline{P X}}{\overline{L_{0} Y}}=\frac{\overline{P L}}{\overline{L_{0} L}}
$$

we find $\delta /\left(-l_{0}\right)=l /\left(l-l_{0}\right)$, which is another form of equation (6).

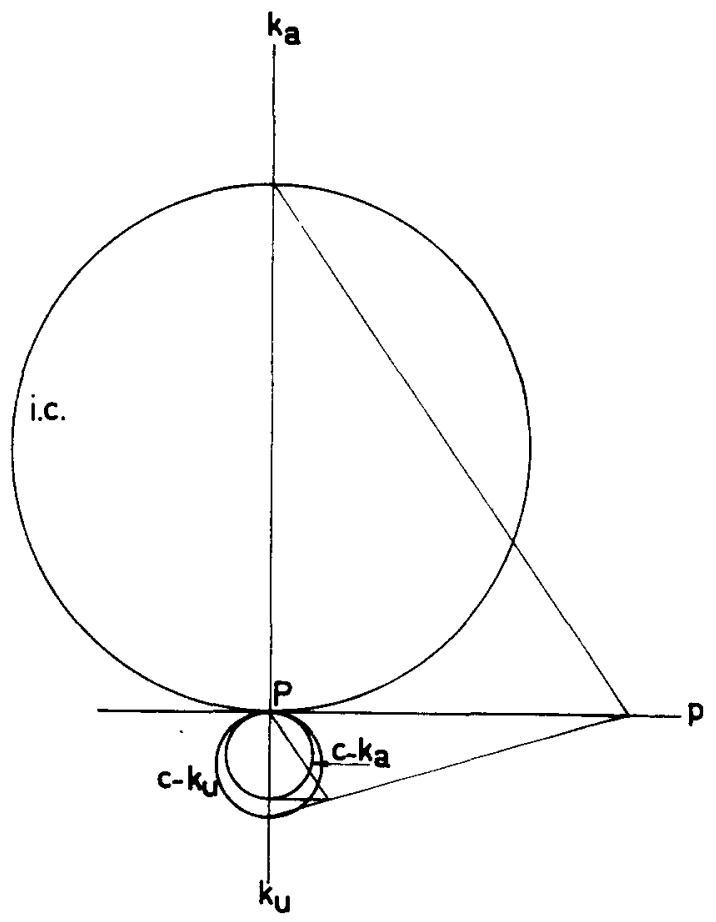

Fig. 4. Pericycloidal position: $R_{0}<R<\frac{5}{4} R_{0},-\delta<l<0,-\frac{1}{2} \delta<l_{0}<0$. 


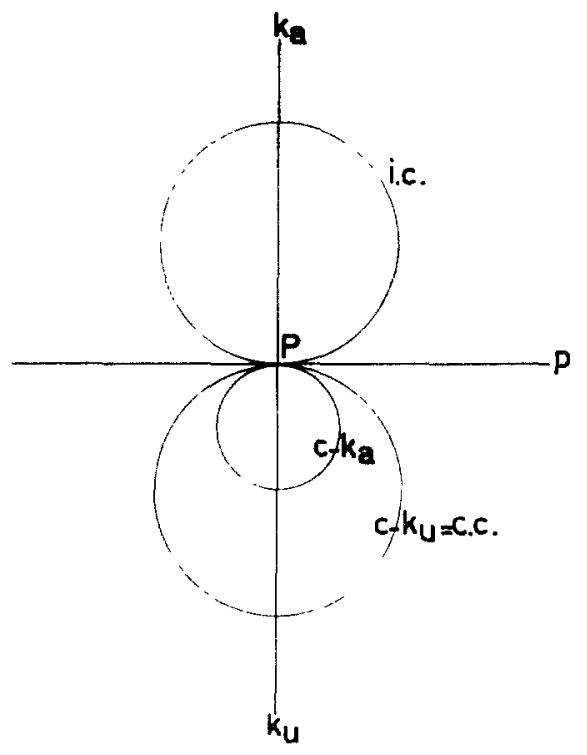

Fro. 5. Pericycloidal position: $R=\frac{5}{4} R_{0}, l=-\delta, l_{0}=-\frac{1}{2} \delta$, c.c. $=$ cuspidal circle $=$ inffection circle of the inverse motion.

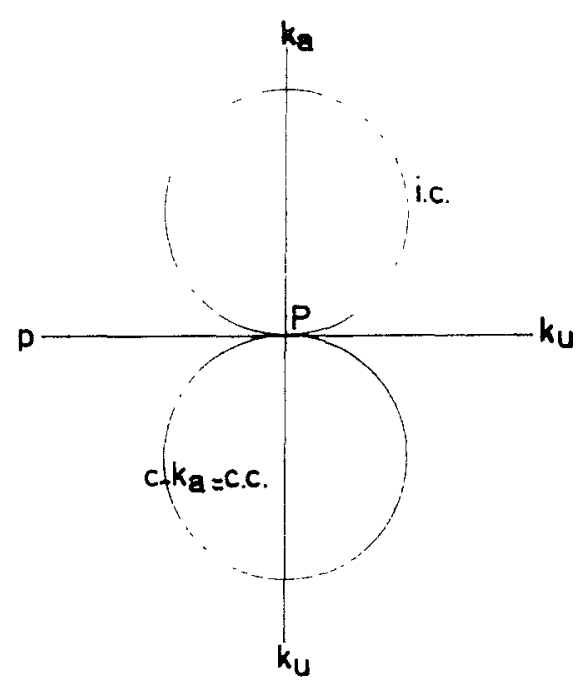

FIG. 6. Cardioidal position (=kinematical inversion of the cardan position): $R=2 R_{0}, l=-\infty, l_{0}=\cdot \delta$. 


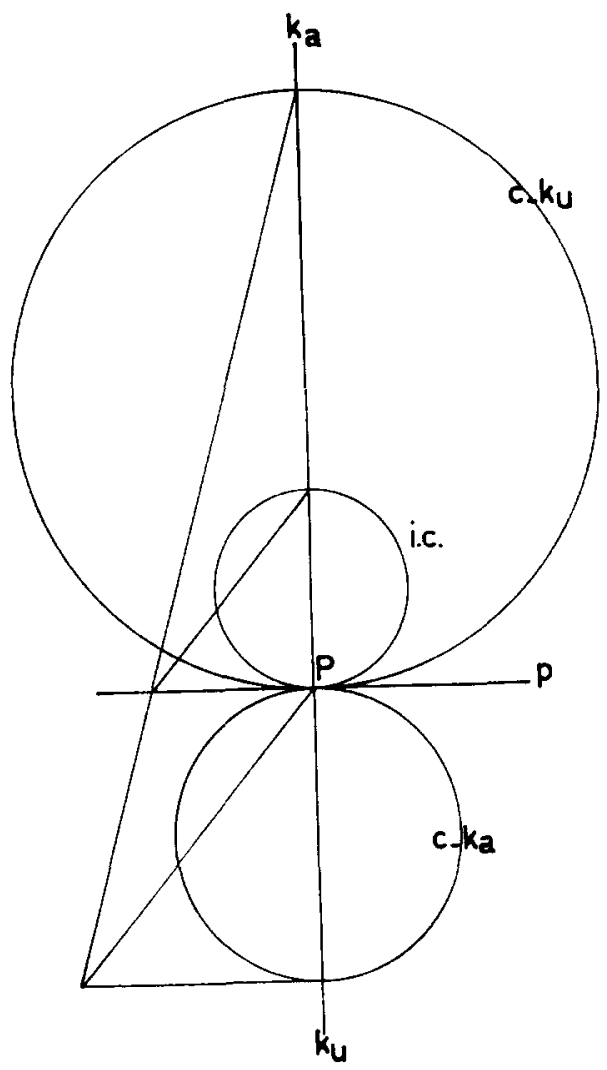

FIG. 7. Evolvent position: $R=\infty, l=3 \delta, l_{0}=-\frac{3}{2} \delta$.

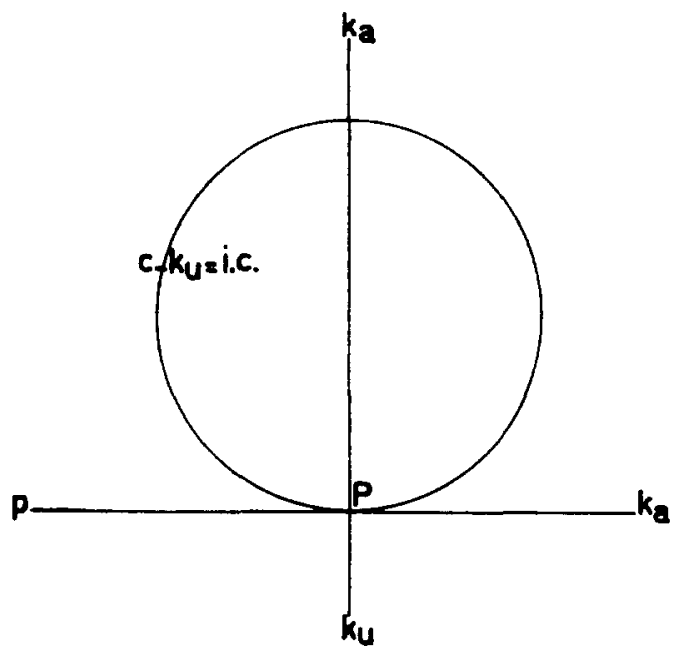

FiG. 8. Elliptical or cardan position: $R=\frac{1}{2} R_{0}, l=\delta, l_{0}=-\infty$. 


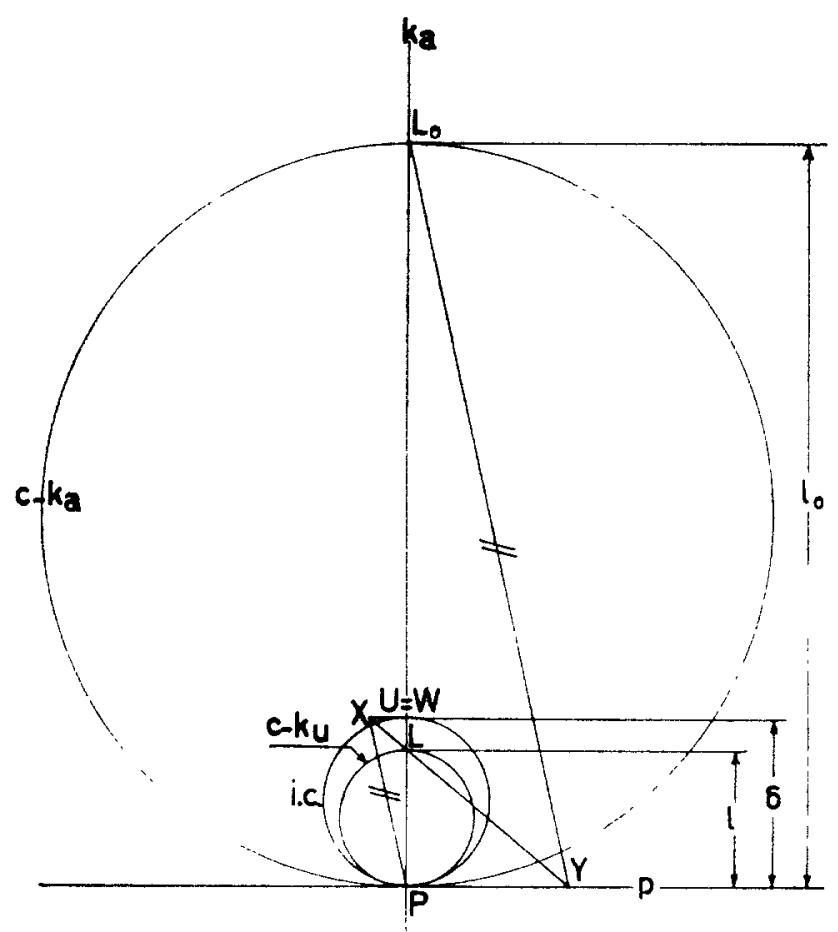

ku

Fro. 9. Hypocycloidal position: $\frac{1}{2} R_{0}<R<\frac{4}{5} R_{0}, \frac{1}{2} \delta<l<\delta, \delta<l_{0}<\infty$.

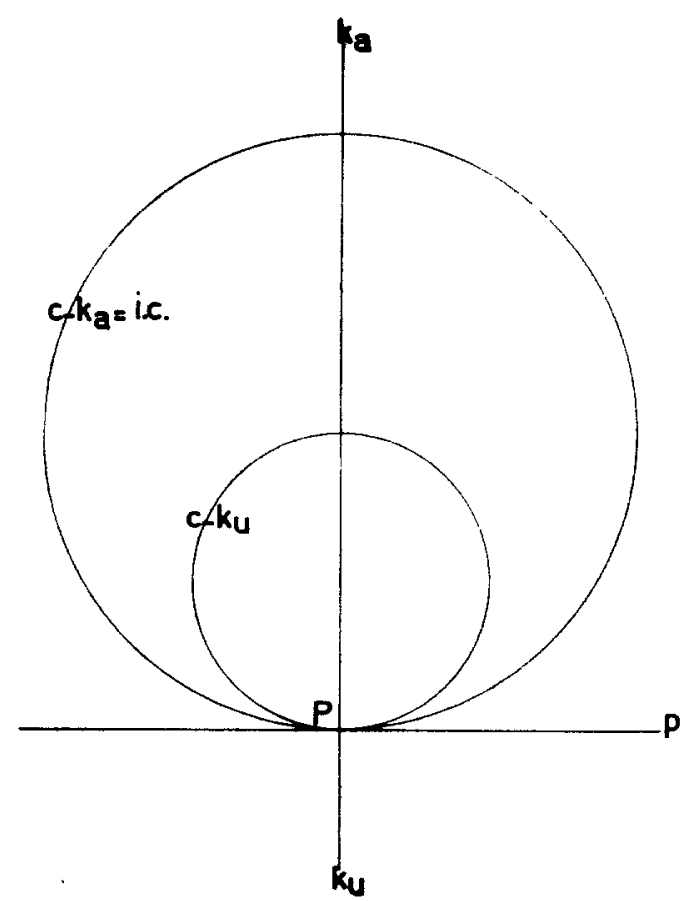

Fro. 10. Hypocycloidal position: $R=\frac{4}{5} R_{0}, l=\frac{1}{2} \delta, l_{0}=\delta$. 


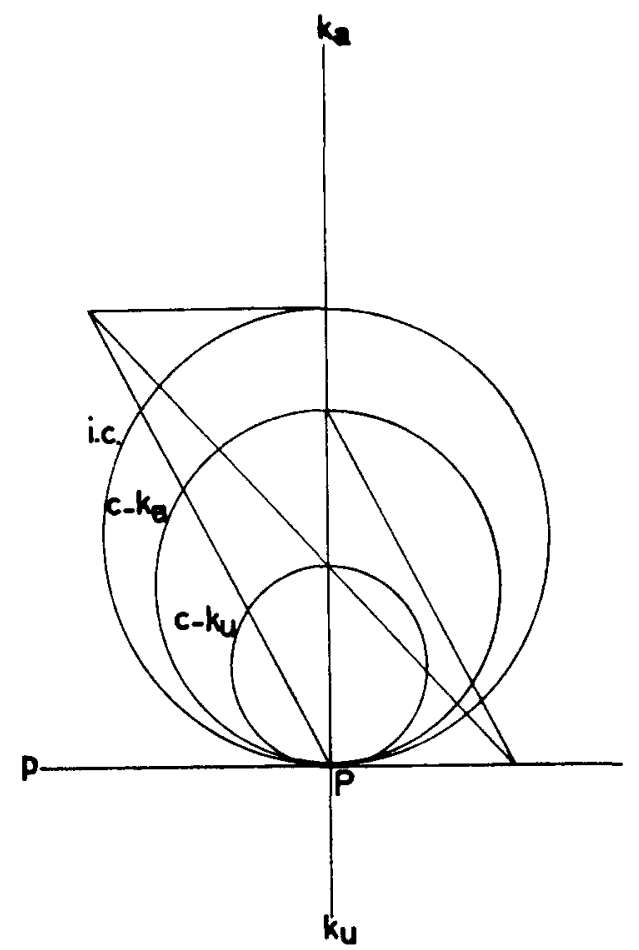

FIG. 11. Hypocycloidal position: $\frac{4}{5} R_{0}<R<R_{0}, 0<l<\frac{1}{2} \delta, 0<l_{0}<\delta$.

The way in which the three interdependent circles increase and decrease is shown in a cycle of Figs. 4 to 11 . Ball's point will here always be found in the inflection pole $W$, as the intersection of the inflection circle and the $k_{u}$ curve.

4.1.1 Design of the driving mechanism. In the degenerated position of the four-bar linkage referred to above, there are three possibilities for assigning the places to the moving turning-points $A$ and $B$ on $k_{u}$ (folding linkages not taken into account).

(a) $B$ on $n$ and $A$ on the circle that is $a$ branch of $k_{u}$. From the equation of Euler-Savary we have $\overline{P B}^{2}=\overline{B B}_{0} . \overline{B B}_{w}$, while $B_{w}=W=U=K$ and also $\overline{B A}=\overline{B B}_{0}=\overline{B K}$.

This leads to $\overrightarrow{P B}^{2}=\overline{B W}^{2}$, so $B$ coincides with the centre of the inflection circle, and $A=P$. From the theorem of Bobillier the centre of curvature corresponding to $A$ lies on $p$. Generally speaking, however, the pole tangent $p$ is not part of curve $k_{a}$. So in general no solution is possible. Only in the special case of a cardan position of the four-bar linkage, that is to say, if $R=\frac{1}{2} R_{0}$ (see Fig. 8), the circle forming part of $k_{a}$ 


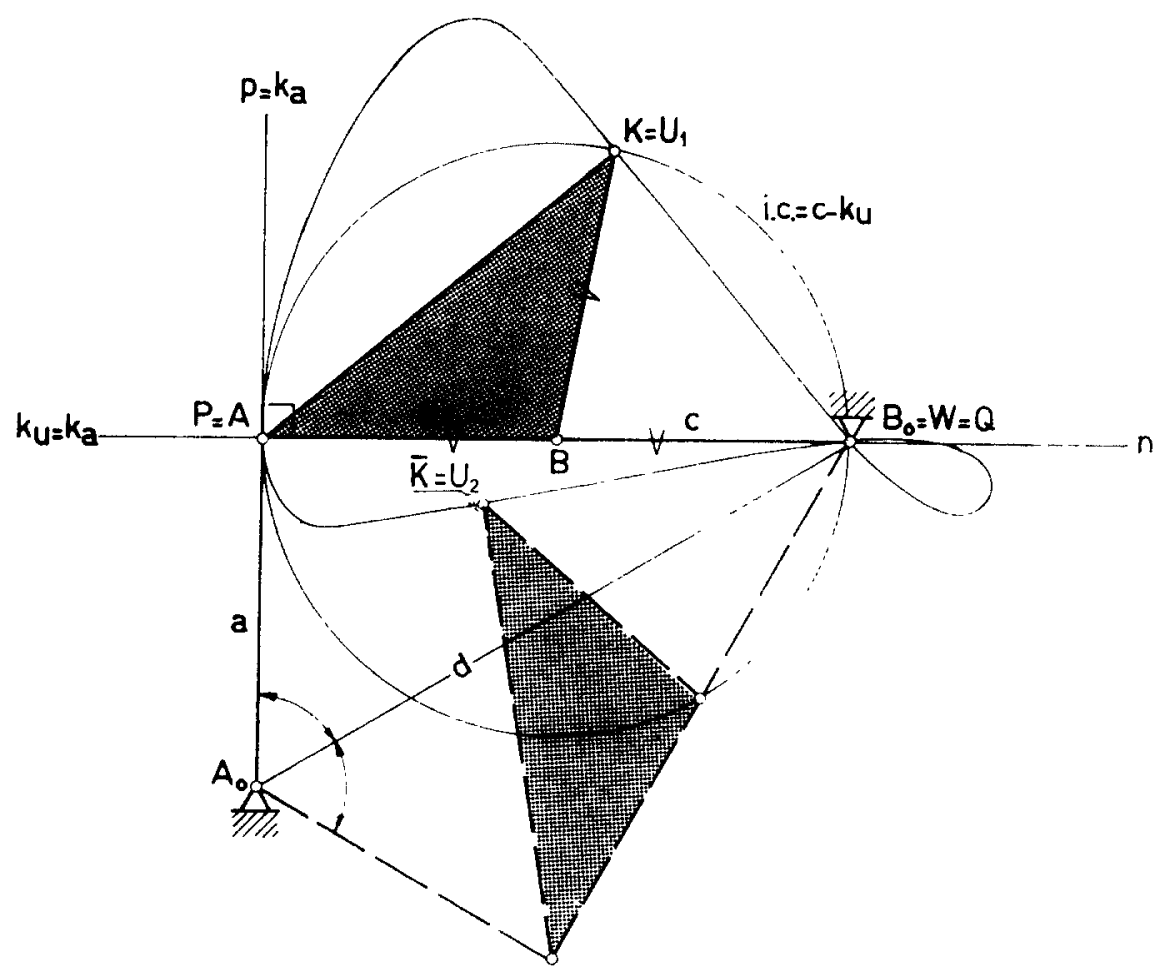

FIG. 12.

is infinitely large. In that case only the pole tangent forms part of $k_{a}$. As also in this case the line at infinity belongs to $k_{a}$, the inflection circle is a branch of $k_{u}$. So any point of the inflection circle is then a Ball's point and may be considered to be a coupler point. The design is shown in Fig. 12. As the quadrilateral does not satisfy the condition of Grashof, this case can be left out of consideration.

(b) A as well as $B$ on the circle that is a branch of $k_{u}$ (see Fig. 13). (Henceforth a circle that is a branch of $k_{u}$ or of $k_{a}$ will be indicated by $c-k_{u}$ and $c-k_{a}$ respectively.)

If $c-k_{u}$ and the inflection circle are lying on opposite sides of $p$, then $\overline{B B}_{0}<\overline{B U}$, and, hence, if $U=K$, $\overline{B B}_{0} \neq \overline{B K}$. Only when $c-k_{u}$ and the inflection circle are on the same side of $p$, that is to say, when $l / \delta>0$, is it possible to find a point $B$, for which $\overrightarrow{B B}_{0}=\overrightarrow{B U}$. Hence in the cycle of Fig. 4 to 11 the degenerated configuration of Fig. 6 is a limiting situation for the solution of our problem. This is also the case with the degenerated configuration of Fig. 10, where $l / l_{0}=\frac{1}{2}$.

Thus real points $B$ can only be found if $\left|l_{0}\right| \geqq \delta$. It is also evident that if $c-k_{u}$ and $c-k_{a}$ are on the same side of $p$, no real points $A$ on $c-k_{u}$ can be found that satisfy the condition $\overline{B A}=\overline{B B}_{0}$. Thus there 


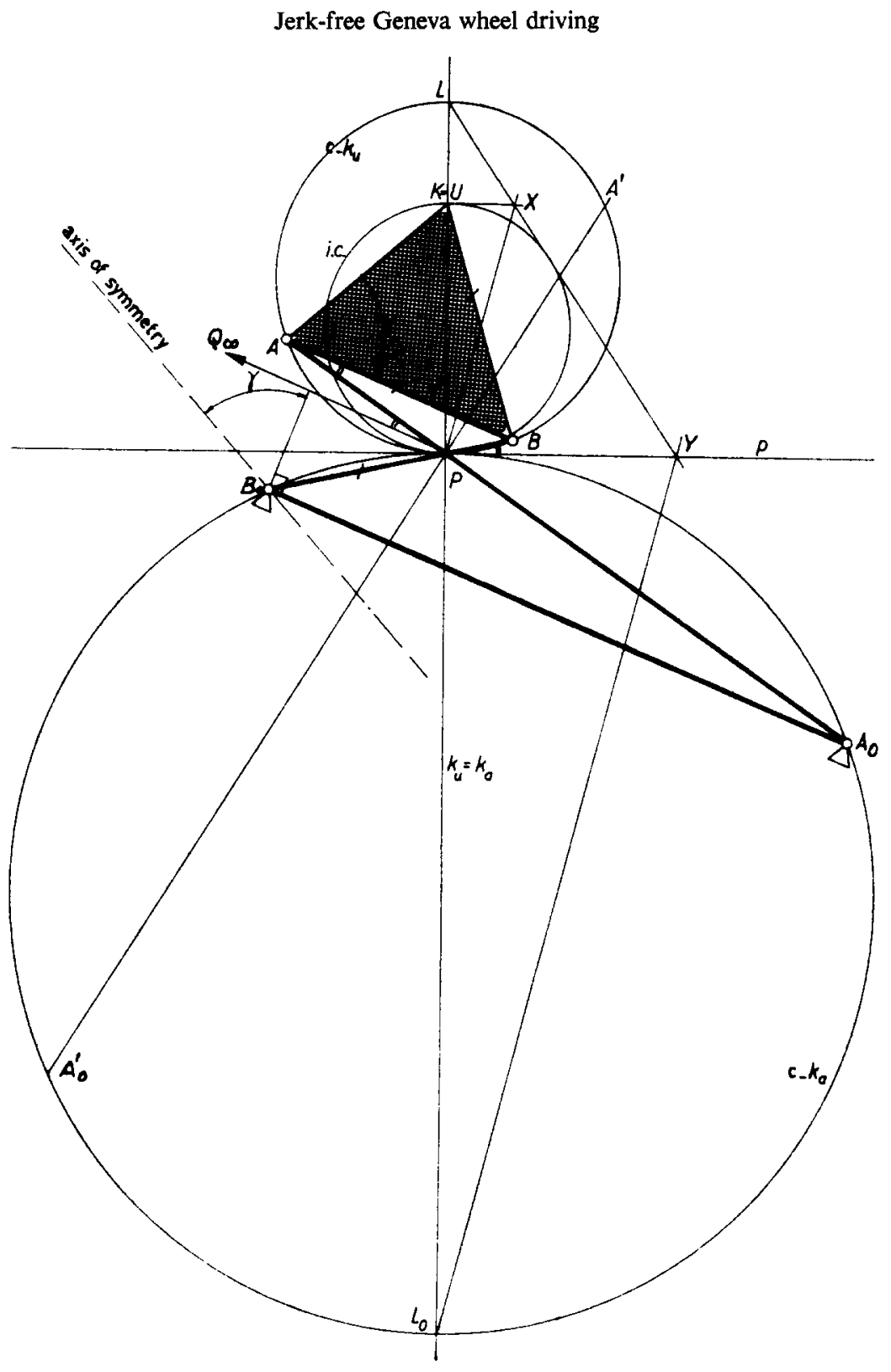

FIG. 13.

are only solutions of the type of Fig. 13, where $c-k_{u}$ and $c-k_{a}$ are on opposite sides of $p$ and the links $A A_{0}$ and $B B_{0}$ cross in $P$.

The coupler curve corresponding to Fig. 13 is shown in Fig. 14. There is a double-point where the path really intersects itself at $\boldsymbol{B}_{\mathbf{0}}$. Furthermore, a four-bar linkage of this type does not satisfy the condition of Grashof. Hence, in this case no solution useful to our purpose can be found. 


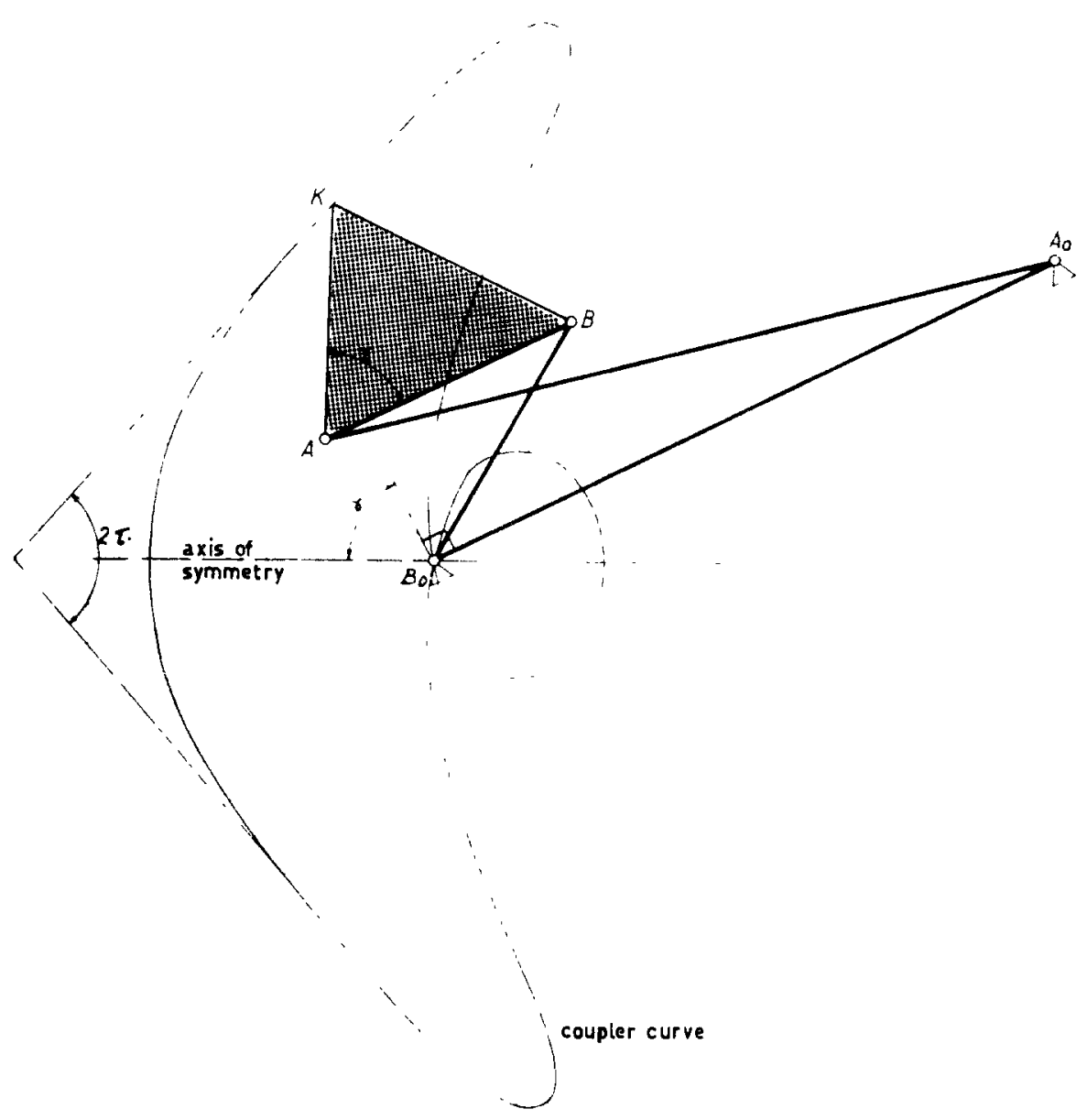

FIG. 14.

(c) B on $c-k_{u}$ and $A$ on $n$, as another branch of $k_{u}$ (see Fig. 15). The construction then is as follows: Dependent on the desired angle $2 \tau$, we start from a ratio $l / l_{0}$, taken from Fig. 16, between the diameters of $c-k_{u}$ and $c-k_{a}$. Just as in case (b) we have $l>0$ and $\left|l_{0}\right| \geqq \delta$. (A negative value of $l_{0}$ means that $c-k_{a}$ and the inflection circle are on opposite sides of $p$.) With the aid of equation (6) we now draw the circles $c-k_{\mathfrak{w}}, c-k_{a}$ and the inflection circle. Moreover, the points $P, B$ and $B_{0}$ lie on one straight line in such a way that $B$ lies on $c-k_{u}$ and $B_{0}$ on $c-k_{a}$. On the assumption that $\overline{B B}_{0}=\overline{B K}$ we have

$$
\left|\frac{\overline{P B}}{\overline{U B}}\right|=\left|\frac{\overline{P B}}{\overline{B K}}\right|=\left|\frac{\overline{P B}}{\overline{B B}_{0}}\right|=\left|\frac{l \sin \varphi}{\left(l_{0}-l\right) \sin \varphi}\right|=\left|\frac{l}{\left(l-l_{0}\right)}\right|=\left|\begin{array}{l}
\delta \\
l_{0}
\end{array}\right| \leqq 1 .
$$




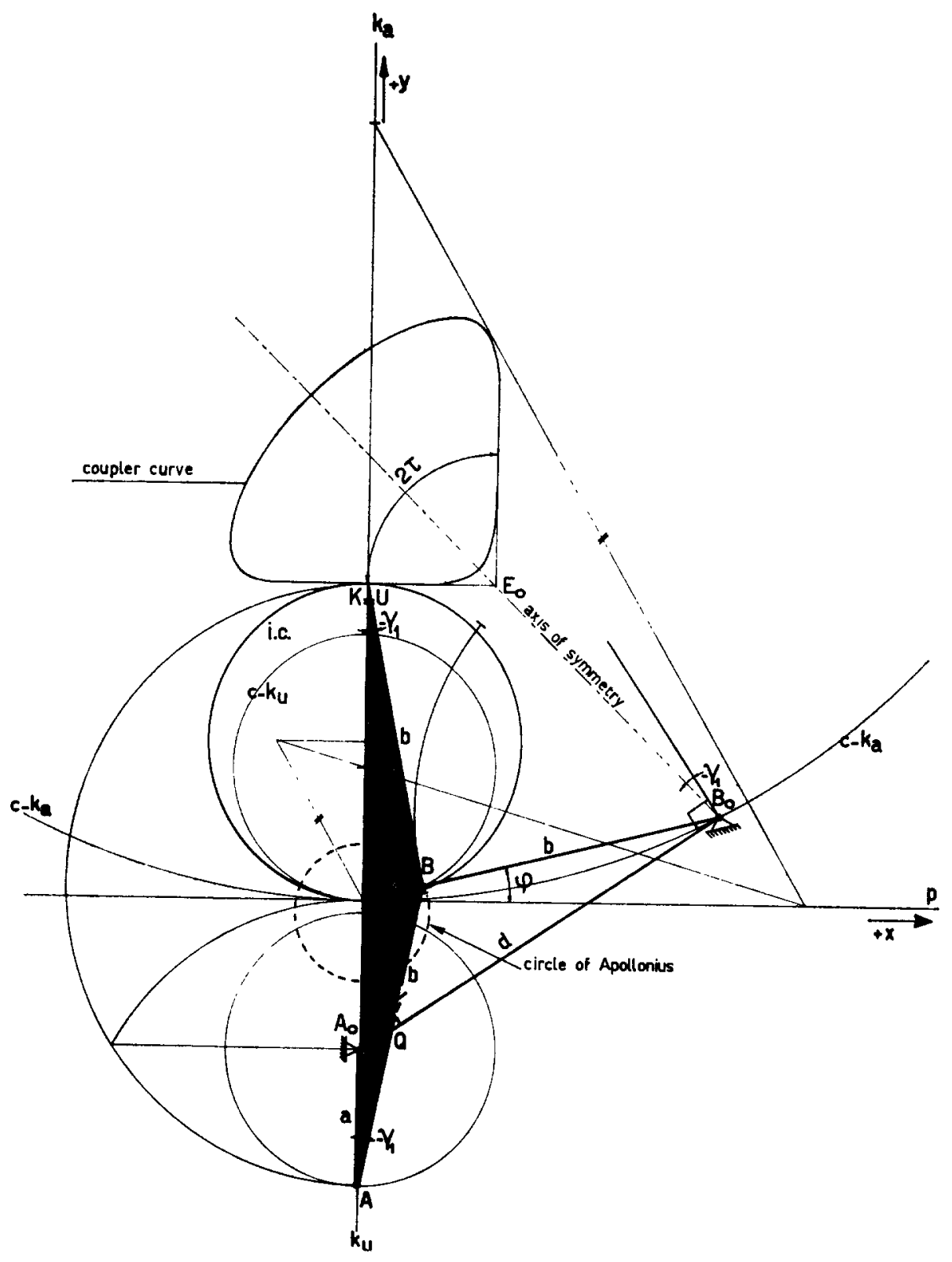

FrG. 15.

Hence, besides $c-k_{\mathrm{u}}$ a second locus can be indicated for point $B$, viz. the locus of those points for which the ratio of the distances to two fixed points $U$ and $P$ is given. This locus is the circle of Apollonius (see Fig. 15). 
E. A. DIJKSMAN

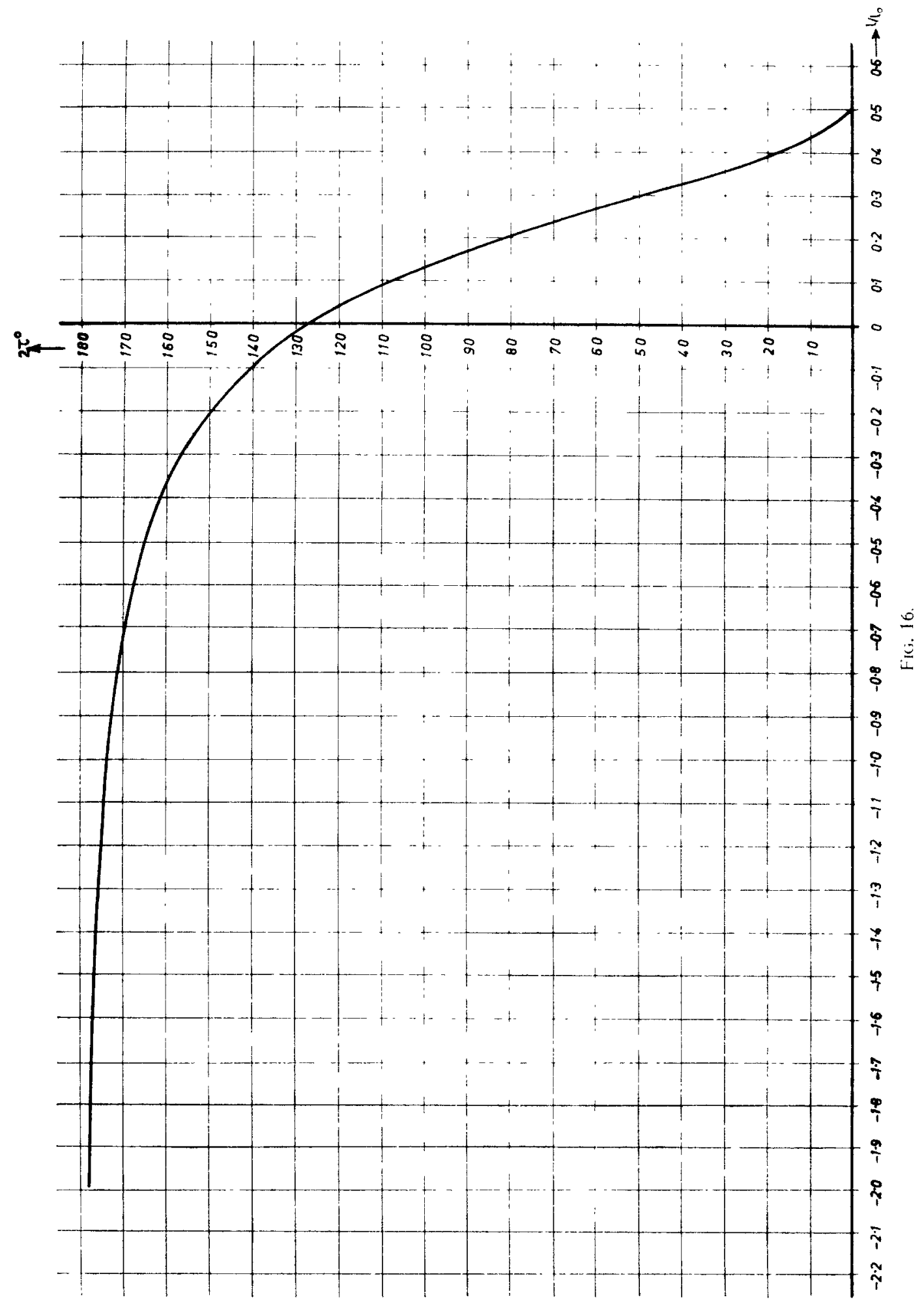


As $\overline{B A}=\overline{B B}_{0}$ the turning-point $A$ is found at one of the two intersections of the pole normal with a circle with centre $B$ and radius $\overrightarrow{B B}_{0}$. One of those intersections exactly coincides with the Ball's point, which can not be taken as turning-point $A$. The fixed point $A_{0}$ lies also on the pole normal and can be found by the equation $\overline{P A}^{2}=\overline{A A}_{0} \cdot \overline{A A}_{\mathrm{w}}$. This follows from the Euler-Savary equation. The point $A_{w}$ coincides with $W, U$ and $K$. The corresponding coupler curve appears to have a path suitable for our purpose.

We are also able to determine the dimensions of the mechanism. We do this by using a number of equations, the derivation of which will, for the sake of brevity, be omitted. With

$$
\begin{aligned}
q & =1-2\left(l / l_{0}\right), \\
\tan ^{2} \tau & =\frac{2 q^{3}\left(q^{2}+1\right)\left(2 q^{2}+q+1\right)^{2}}{\left(3 q^{2}+1\right)^{2}}
\end{aligned}
$$

Moreover,

$$
\frac{a}{b}=\frac{2 q^{2}}{3 q^{2}+1}\left(\frac{q+1}{2 q^{2}-q+1}\right)^{\frac{1}{2}}
$$

and

$$
\frac{d}{b}=\frac{2}{3 q^{2}+1}\left(\frac{2 q^{4}+2 q^{3}+4 q^{2}+q+1}{q+1}\right)^{\frac{1}{2}}
$$

while

$$
\tan \gamma_{1}=\frac{q-1}{3 q^{2}+1}\left(2 q\left(q^{2}+1\right)\right)^{\frac{1}{2}}
$$

Finally

$$
\frac{\bar{B}_{0} E_{0}}{b}=\frac{2}{(q+1)\left(2 q^{2}+q+1\right)}\left(\frac{2 q\left(2 q^{2}-q+1\right)\left(2 q^{4}+2 q^{3}+4 q^{2}+q+1\right)}{q^{2}+1}\right)^{\frac{1}{2}}
$$

and

$$
\frac{\overline{E_{0} U}}{b}=\frac{2 q}{2 q^{2}+q+1}\left(\frac{2 q\left(2 q^{2}-q+1\right)}{(q+1)\left(q^{2}+1\right)}\right)^{\frac{1}{2}}
$$

For a number of angles $2 \tau$, the results are given in Table 1 .

\subsection{The cardan position of the four-bar linkage}

When in addition to $\mathrm{d} \delta / \mathrm{d} s=0$ (or $m^{-1}=0$ ) also $R_{0}=2 R$ (or $l_{0}^{-1}=0$ ), the circling-point curve $k_{u}$ is degenerated into the inflection circle and the pole normal. Then the centeringpoint curve $k_{a}$ is degenerated in the pole tangent, in the pole normal and in the line at infinity (see Fig. 8). The cardan position may be presented as a special case of the general cycloidal position, as treated in section 4.1. Choosing the coupler point $K$ also in the inflection pole $W$ of the cardan position, we then have only one angle $2 \tau$, for which the problem can be solved. In the cardan position however, the inflection circle forms part of $k_{u}$. 


\begin{tabular}{|c|c|c|c|c|c|c|c|c|}
\hline$\stackrel{\circ}{\mathscr{O}}$ & 0 & 0 & : & $\therefore$ & 0 & 0 & $\stackrel{8}{i}$ & 8 \\
\hline$\stackrel{i}{i}$ & 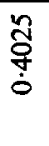 & $\underset{\stackrel{\leftrightarrow}{*}}{\stackrel{\leftrightarrow}{0}}$ & $\frac{\stackrel{0}{o}}{\frac{\dot{q}}{+}}$ & 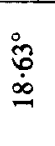 & 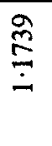 & \begin{tabular}{l}
8 \\
8 \\
\hdashline \\
$\vdots$ \\
0
\end{tabular} & $\begin{array}{l}\infty \\
\stackrel{\infty}{\circ} \\
\stackrel{\circ}{0} \\
\hat{i}\end{array}$ & 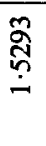 \\
\hline 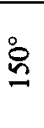 & \begin{tabular}{l}
$\infty$ \\
$\stackrel{2}{+}$ \\
\multirow{+}{0}{} \\
0
\end{tabular} & 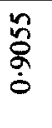 & $\begin{array}{l}\stackrel{0}{0} \\
\dot{q} \\
+\end{array}$ & $\stackrel{\circ}{\stackrel{i}{a}}$ & $\begin{array}{l}\stackrel{0}{0} \\
\stackrel{0}{ \pm}\end{array}$ & $\begin{array}{l}\stackrel{\infty}{त} \\
\stackrel{n}{0} \\
\stackrel{0}{0}\end{array}$ & 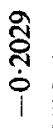 & $\underset{\stackrel{N}{*}}{\stackrel{N}{-}}$ \\
\hline$\stackrel{i n}{=}$ & $\begin{array}{l}\stackrel{\circ}{\circ} \\
\stackrel{2}{+} \\
\dot{0}\end{array}$ & $\begin{array}{l}\widetilde{\infty} \\
\stackrel{\infty}{0} \\
\stackrel{D}{-}\end{array}$ & $\begin{array}{c}\stackrel{0}{0} \\
\stackrel{m}{+}\end{array}$ & 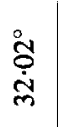 & 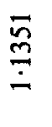 & $\begin{array}{l}\stackrel{\Xi}{\Xi} \\
\dot{0}\end{array}$ & $\begin{array}{l}8 \\
8 \\
0 \\
0 \\
1\end{array}$ & $\begin{array}{l}\tilde{n} \\
\stackrel{n}{-}\end{array}$ \\
\hline 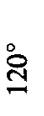 & 号 & $\frac{⿱ 乛 ⿻}{ \pm}$ & 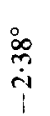 & $\begin{array}{l}\stackrel{i n}{n} \\
\stackrel{n}{n}\end{array}$ & $\begin{array}{l}\stackrel{9}{8} \\
\stackrel{0}{\dot{0}}\end{array}$ & 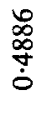 & $\begin{array}{l}\infty \\
2 \\
0 \\
0 \\
\dot{\varphi} \\
+\end{array}$ & $\begin{array}{l}\overline{\overline{0}} \\
\stackrel{0}{0} \\
\dot{0}\end{array}$ \\
\hline in & $\begin{array}{l}\stackrel{\infty}{\infty} \\
\stackrel{\infty}{*} \\
0\end{array}$ & $\stackrel{\frac{n}{9}}{\stackrel{\Upsilon}{-}}$ & $\stackrel{\circ}{\dddot{i}}$ & $\begin{array}{l}\text { in } \\
\text { í }\end{array}$ & 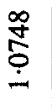 & $\begin{array}{l}8 \\
\stackrel{0}{0} \\
+ \\
0\end{array}$ & $\stackrel{m}{\Xi}$ & $\begin{array}{l}\frac{}{5} \\
\dot{0} \\
\dot{0}\end{array}$ \\
\hline$\&$ & \begin{tabular}{l}
\multirow{N}{J}{} \\
$\dot{0}$
\end{tabular} & 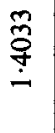 & 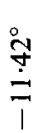 & 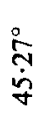 & \begin{tabular}{l}
$\infty$ \\
$\infty$ \\
\multirow{0}{0}{} \\
$\stackrel{\Xi}{\mid}$
\end{tabular} & 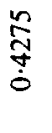 & $\begin{array}{l}\frac{ \pm}{0} \\
\frac{0}{0}\end{array}$ & $\begin{array}{l}0 \\
\text { 芒 } \\
\infty \\
0 \\
0\end{array}$ \\
\hline 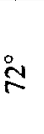 & $\frac{\mathfrak{\sigma}}{\hat{\sigma}}$ & 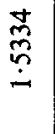 & $\begin{array}{l}\stackrel{0}{\infty} \\
\stackrel{0}{\dot{0}} \\
\stackrel{1}{1}\end{array}$ & $\begin{array}{l}\stackrel{a}{a} \\
\dot{m}\end{array}$ & $\stackrel{ }{\stackrel{2}{\grave{g}}}$ & 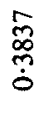 & 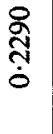 & 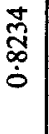 \\
\hline 8 & $\begin{array}{l}\stackrel{\delta}{0} \\
\stackrel{N}{0} \\
\dot{0}\end{array}$ & $\begin{array}{l}\infty \\
\frac{\infty}{6} \\
\dot{-}\end{array}$ & $\begin{array}{l}\dot{\infty} \\
\infty \\
\dot{\infty} \\
\stackrel{1}{1}\end{array}$ & $\begin{array}{l}\infty \\
\stackrel{9}{0} \\
\dot{i}\end{array}$ & 品 & 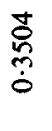 & 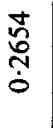 & $\begin{array}{l}\stackrel{\rho}{m} \\
\stackrel{\infty}{0} \\
\dot{0}\end{array}$ \\
\hline 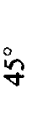 & $\begin{array}{c}\tilde{\alpha} \\
\dot{\alpha} \\
\dot{\sigma}\end{array}$ & $\underset{\Xi}{\stackrel{\Xi}{\Xi}}$ & 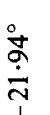 & $\stackrel{\circ}{\stackrel{\infty}{\infty}}$ & $\begin{array}{l}\overline{\mathscr{\alpha}} \\
\stackrel{\alpha}{0} \\
\dot{0}\end{array}$ & 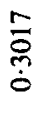 & $\begin{array}{l}n \\
\hat{\sigma} \\
\dot{o} \\
\dot{0}\end{array}$ & $\begin{array}{l}n \\
\infty \\
0 \\
0\end{array}$ \\
\hline 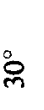 & $\frac{\text { त్రి }}{\dot{0}}$ & 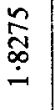 & $\begin{array}{l}\stackrel{\vec{T}}{\dot{\sim}} \\
\dot{\sim}\end{array}$ & $\stackrel{\stackrel{i}{a}}{\grave{g}}$ & 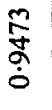 & $\underset{\stackrel{\stackrel{P}{+}}{0}}{\dot{\sigma}}$ & $\begin{array}{l}\text { के } \\
\hat{\sigma} \\
\hat{0}\end{array}$ & $\begin{array}{l}\tilde{a} \\
\underset{\infty}{\infty} \\
\dot{0}\end{array}$ \\
\hline in & 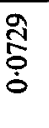 & 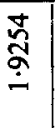 & 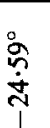 & $\begin{array}{l}\stackrel{\circ}{6} \\
\dot{+}\end{array}$ & $\begin{array}{l}\sqrt[5]{0} \\
\infty \\
0 \\
0\end{array}$ & $\frac{\tilde{n}}{\dot{0}}$ & $\begin{array}{l}0 \\
\vdots \\
\vdots \\
\vdots \\
0\end{array}$ & $\begin{array}{l}0 \\
\stackrel{0}{0} \\
0 \\
0 \\
0\end{array}$ \\
\hline : & 0 & $N$ & : & : & 0 & 0 & $+\infty$ & - \\
\hline$\stackrel{5}{N}$ & $\frac{0}{8}$ & $\frac{0}{8}$ & 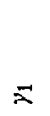 & 몈 & 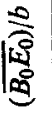 & 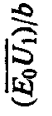 & $\stackrel{\circ}{\Sigma}$ & 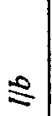 \\
\hline
\end{tabular}


So besides $W$, any point of the inflection circle is a Ball's point. Hence the whole inflection circle is at our disposal for choosing coupler point $K$, which means that, in the cardan position, any angle $2 \tau$ can be realised by means of a four-bar linkage.

4.2.1 Design of the driving mechanism. Restricting ourselves to finite lengths of the links, the turning-points $A$ and $B$ can not be chosen on the inflection circle. Point $P$ is an exception to this rule, for in this point the radius of curvature of the path may take any value between 0 and $\infty$. By not choosing $A$ or $B$ in $P$ we get a folding linkage, in which all links are superimposed, and the minimum transmission angle $\mu_{\min }=0$. By choosing $A$ in $P$ we get the mechanism of Fig. 12, which has already been discussed in part (a) of section 4.1.1.

If $B$ and $P$ coincide, the construction is as follows (see Fig. 17):

(a) Draw the inflection circle i.c., the pole tangent $p$ and pole normal $P W$.

(b) Determine angle $\gamma_{1}$ in Fig. 18 at a given angle $2 \tau$. (For instance if $2 \tau=90^{\circ}$ then $\gamma_{1}=16.47^{\circ}$.)

(c) Take $\Varangle W P K=2\left|\gamma_{1}\right|$. Choose one of the two possible positions of the free side of this angle. Let $K$ be the point of intersection of this side with the inflection circle.

(d) Draw a circle with centre $P=B$ and radius $\overline{B K}=\overline{B B}_{0}=\overline{B A}$.

(e) Let $A$ be the point of intersection of this circle with the pole normal (n) outside the inflection circle.

(f) Let $B_{0}$ be that point of intersection of the circle mentioned under (d) with $p$ that lies on the same side of $n$ as $K$ if $\gamma_{1}>0$, and on the opposite side of $n$ if $\gamma_{1}<0$.

(g) Determine the fixed turning-point $A_{0}$ with the aid of the equation $\overline{P A}^{2}=\overline{A A}_{w} \cdot \overline{A A}_{0}$, where $A_{w}=W$. (The points $A_{w}$ and $A_{0}$ always lie on the same half of the path normal with respect to turning-point $A$.)

The coupler curve, traced by the coupler point of a four-bar linkage, constructed as described above, turns out to have a suitable shape in all cases. The choice of point $A$ inside the inflection circle leads to a less suitable coupler curve and to a smaller minimum transmission angle $\mu_{\min }$; while the angles $2 \tau$ between $30^{\circ} \cdot 73^{\circ}$ and $178 \cdot 2^{\circ}$ can not be realised.

In connection with the described design we can derive a number of equations with which the dimensions of the driving mechanism and the driven Geneva wheel can be determined. They are:

$$
\begin{gathered}
1+\cos 2 \gamma_{1}=\tan \left(\tau+\gamma_{1}\right) \\
\frac{a}{b}=\frac{\cos 2 \gamma_{1}}{1+\cos 2 \gamma_{1}} \\
\frac{d}{b}=\frac{1}{\sin \left(\tau+\gamma_{1}\right)} \\
\frac{\bar{B}_{0} E_{0}}{b}=\frac{1-\sin 2 \gamma_{1}}{\sin \tau}
\end{gathered}
$$




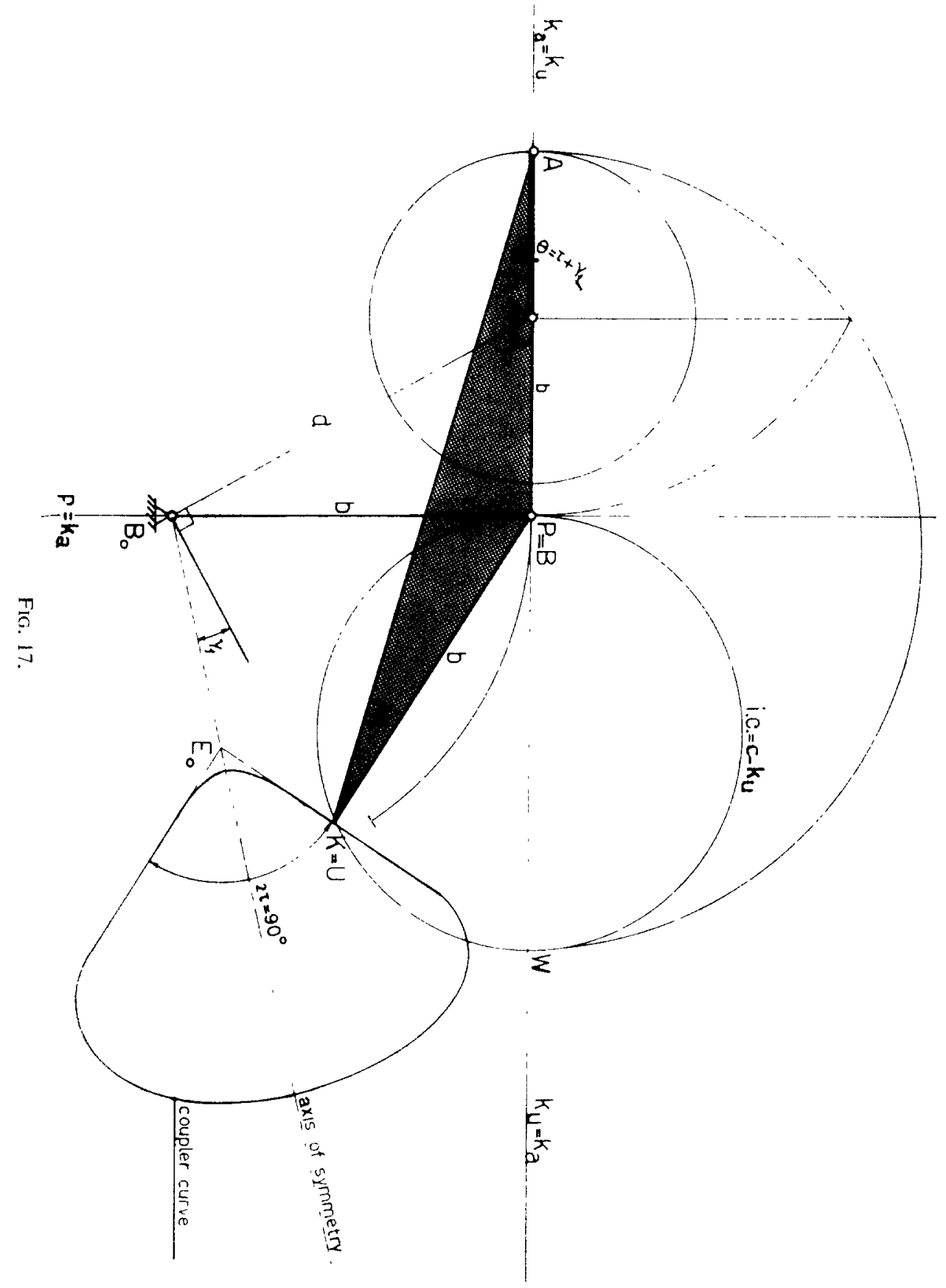

NWWSYIIT $\mathrm{V} \cdot \mathrm{H}$ 


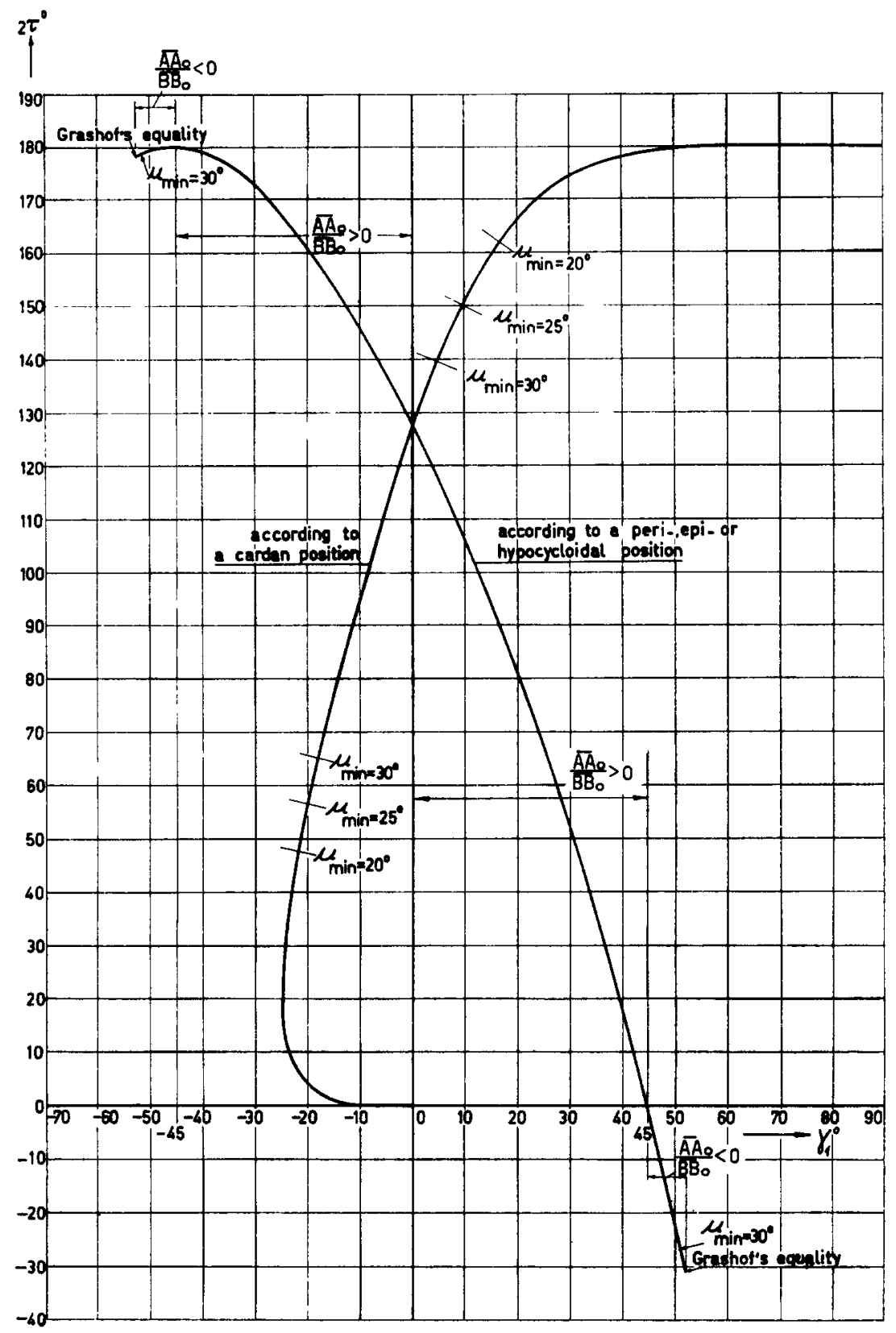

FIG. 18. 


$$
\frac{\overline{E_{0} U}}{b}=\left|\cos 2 \gamma_{1}-\frac{1-\sin 2 \gamma_{1}}{\tan \tau}\right|
$$

It may be seen that always

$$
\mu_{\min } \geqq 2 \arcsin \frac{1}{4}\left(5^{\frac{1}{2}}-1\right) \approx 36^{\circ},
$$

which is a reasonable and permissible angle.

It will be clear that only when $\gamma_{1}=0$ does the coupler point $K$ coincide with the inflection pole $W$. This will be the case when $2 \tau=2 \arctan 2=126 \cdot 87^{\circ}$. Only then is the solution according to the general cycloidal position identical with the one according to the cardan position. For significant values of $2 \tau$ the results according to the cardan position are given in Table 2.

\subsection{Degeneration of the $k_{u}$ curve, when $l^{-1}=0$ (or $R=2 R_{0}$ )}

If $l^{-1}=0$, it follows from equation (1) that either $\varphi=0$, which is the equation of $p$, or $r=m \cos \varphi$, which is the equation of a circle through $P$ with its centre on $p$. Hence, the $k_{u}$ curve degenerates into $p$ and a circle through $P(0,0)$ and $M(m, 0)$ with its centre on $p$. The $k_{a}$ curve keeps its general shape. The circle that is a branch of $k_{u}$, and the inflection circle intersect at the pole and at the only Ball's point.

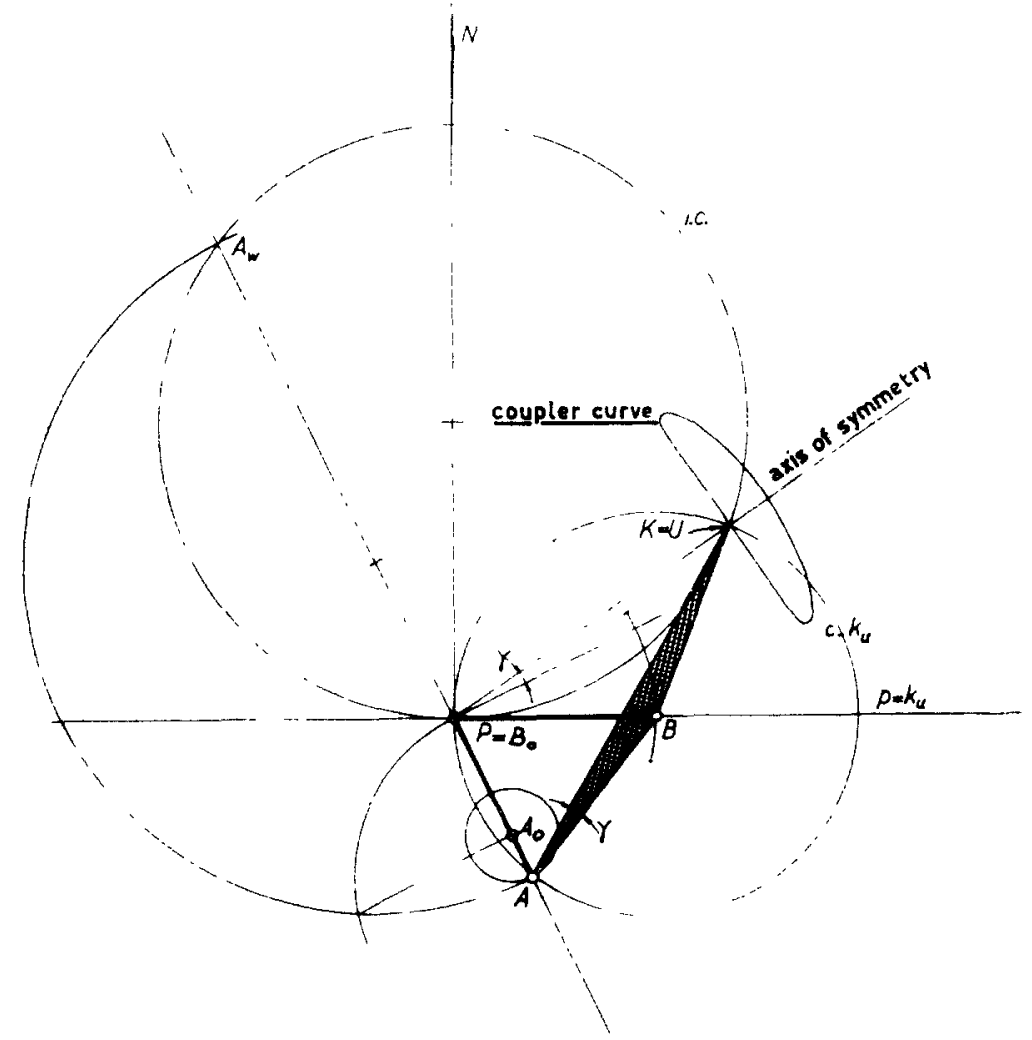

FIG. 19. 


\begin{tabular}{|c|c|c|c|c|c|c|c|c|c|c|c|}
\hline$\stackrel{\circ}{\circ}$ & $\frac{9}{9}$ & 0 & $\vec{\sim}$ & $\pi$ & $\mathrm{N}$ & 0 & \& & \& & ; & 0.0 & 0 \\
\hline$\stackrel{\overbrace{}}{\varrho}$ & 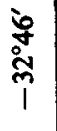 & 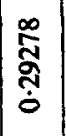 & \begin{tabular}{l} 
\& \\
\multirow{ָ}{*}{} \\
-
\end{tabular} & 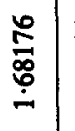 & ষ্ণ & 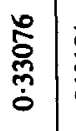 & $\frac{\infty}{\frac{\infty}{\infty}}$ & 窟 & 旁 & & 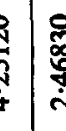 \\
\hline 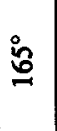 & 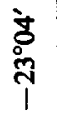 & $\mid \begin{array}{l}0 \\
\vdots \\
\vdots \\
o \\
0\end{array}$ & 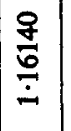 & 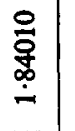 & 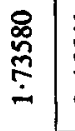 & 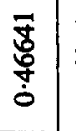 & 商 & 它 & 亏ัे & $\begin{array}{c}\tilde{z} \\
\bar{z} \\
\delta\end{array}$ & 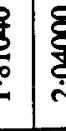 \\
\hline$\stackrel{\circ}{\circ}$ & 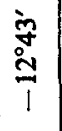 & 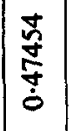 & 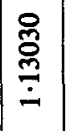 & $\begin{array}{l}\text { 臺 } \\
\stackrel{-1}{*}\end{array}$ & 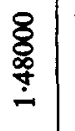 & $\begin{array}{l}\text { प0 } \\
0 \\
0\end{array}$ & ¿̊̀ & 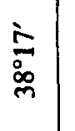 & 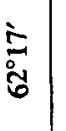 & 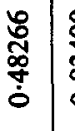 & 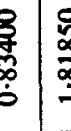 \\
\hline$\stackrel{\text { in }}{m}$ & 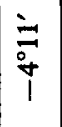 & 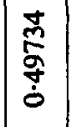 & $\mid \begin{array}{l}\Omega \\
\stackrel{\Xi}{\Xi}\end{array}$ & $\left|\begin{array}{l}\infty \\
\vdots \\
\vdots \\
- \\
-1\end{array}\right|$ & 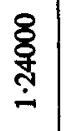 & 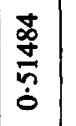 & ذे ڤે & స్ন & & 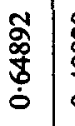 & 总 \\
\hline בे & 莡 & $\mid \begin{array}{l}\infty \\
\tilde{\omega} \\
\sigma \\
0\end{array}$ & $\left|\begin{array}{l}\overrightarrow{\mathbf{D}} \\
\overrightarrow{\underline{z}}\end{array}\right|$ & $\mid \begin{array}{l}\infty \\
0 \\
0 \\
\vdots \\
-\end{array}$ & $\begin{array}{l}\infty \\
\stackrel{\infty}{0} \\
\stackrel{0}{0}\end{array}$ & $\mid \begin{array}{l}\tilde{z} \\
\tilde{o} \\
\tilde{o} \\
0\end{array}$ & : & 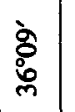 & 岕 & 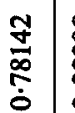 & 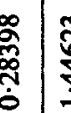 \\
\hline \& & 容 & 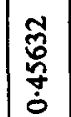 & 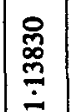 & $\mid$\begin{tabular}{l}
0 \\
$\vdots$ \\
$\vdots$ \\
\hdashline
\end{tabular} & $\mid \begin{array}{l}0 \\
0 \\
0 \\
0\end{array}$ & $\left|\begin{array}{l}0 \\
0 \\
0 \\
0 \\
0\end{array}\right|$ & 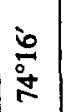 & స్̃ે & 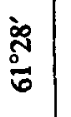 & $\begin{array}{l}\text { : } \\
\text { ợ }\end{array}$ & స్t \\
\hline$\stackrel{乛}{*}$ & 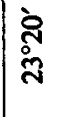 & $\mid \begin{array}{l}\hat{\alpha} \\
\text { 客 } \\
\dot{0}\end{array}$ & 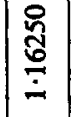 & 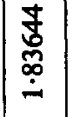 & $\left|\begin{array}{|c}n \\
\frac{n}{0} \\
\vdots \\
0\end{array}\right|$ & $\begin{array}{l}\stackrel{m}{\Xi} \\
\dot{p} \\
\dot{0}\end{array}$ & $\begin{array}{c}\dot{D}^{\circ} \\
\vdots \\
\vdots \\
0\end{array}$ & $\mid \begin{array}{c}\dot{J} \\
\dot{z} \\
\dot{y}\end{array}$ & ڤั & 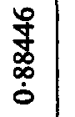 & $\tilde{z}$ \\
\hline \&8 & 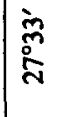 & 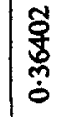 & $\mid \begin{array}{c}0 \\
\stackrel{0}{\infty} \\
\stackrel{\sim}{二}\end{array}$ & 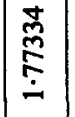 & 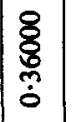 & $\mid$\begin{tabular}{l}
$\overrightarrow{0}$ \\
\multirow{0}{0}{} \\
$\dot{0}$
\end{tabular} & $\mid \begin{array}{c}\text { ò } \\
\text { on } \\
\text { o. }\end{array}$ & $\left|\begin{array}{c}0 \\
\vdots \\
\vdots \\
o \\
o\end{array}\right|$ & $\begin{array}{c}\grave{m} \\
\vdots \\
\vdots \\
\vdots\end{array}$ & 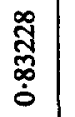 & 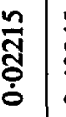 \\
\hline 洛 & $\mid \begin{array}{c}\text { : } \\
\text { స్ల } \\
\text {. }\end{array}$ & 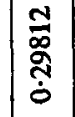 & 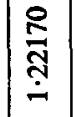 & $\mid \begin{array}{l}\text { 总 } \\
\text { : } \\
\stackrel{0}{-}\end{array}$ & 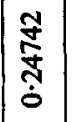 & $\mid \begin{array}{l}n \\
0 \\
0 \\
0\end{array}$ & $\begin{array}{l}\dot{0} \\
\vdots \\
0 \\
\infty\end{array}$ & $\begin{array}{l}8 \\
0 \\
i \\
n\end{array}$ & 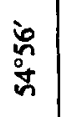 & $\begin{array}{l}\mathbf{t} \\
\stackrel{2}{2} \\
\hat{0}\end{array}$ & 通 \\
\hline 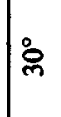 & 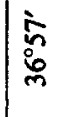 & 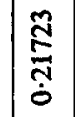 & 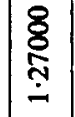 & 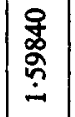 & $\mid \begin{array}{l}0 \\
\tilde{n} \\
0 \\
0\end{array}$ & $\begin{array}{l}\frac{\hat{v}}{\mathrm{~m}} \\
\stackrel{\mathrm{j}}{0}\end{array}$ & 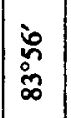 & స్ల్ & $\frac{5}{n}$ & 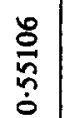 & ఫ్ర్రి \\
\hline in & 产 & $\frac{\tilde{\alpha}}{\tilde{\sigma}}$ & 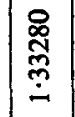 & $\mid \begin{array}{l} \pm \\
6 \\
0 \\
:\end{array}$ & 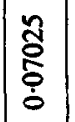 & 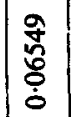 & $\begin{array}{l}\dot{y} \\
0 \\
0 \\
\infty\end{array}$ & 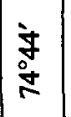 & 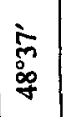 & $\begin{array}{l}\infty \\
\stackrel{\infty}{0} \\
\stackrel{0}{0} \\
\dot{0}\end{array}$ & סे \\
\hline$\therefore$ & is & 0 & $\bar{\approx}$ & $\approx$ & 10 & 0 & \& & : & in & 0 & 0 \\
\hline$\sigma$ & & $\frac{\circ}{8}$ & 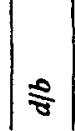 & 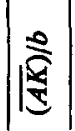 & $\mid$ & 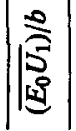 & $1=1$ & 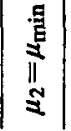 & & & 3 \\
\hline
\end{tabular}


4.3.1 The four-bar linkage in the position, where $R=2 R_{0}$. In this degenerated position of the four-bar linkage there are three possibilities for placing $A$ and $B$ on $k_{u}$.

(a) $B$ on $p$ and $A$ on $c-k_{u}$. From the theorem of Hartmann it follows that $B_{0}=P$. As. in addition, $\overrightarrow{B A}=\overrightarrow{B B}_{0}=\overrightarrow{B K}$, point $B$ can only be taken in the centre of $c-k_{u}$ (see Fig. 19).

Point $A$ can be chosen freely on $c-k_{u}$, while the centre of curvature $A_{0}$ corresponding to $A$ is given by the equation $\overline{P A}^{2}=\overline{A A}_{w}, \overline{A A}_{0}$. Since the coupler point $K$ is a Ball's point, this point turns out to lie exactly on the axis of symmetry of the coupler curve. This means that in any case $2 \tau=180^{\circ}$. Hence the mechanism can be applied to drive a two station Geneva wheel only [1].

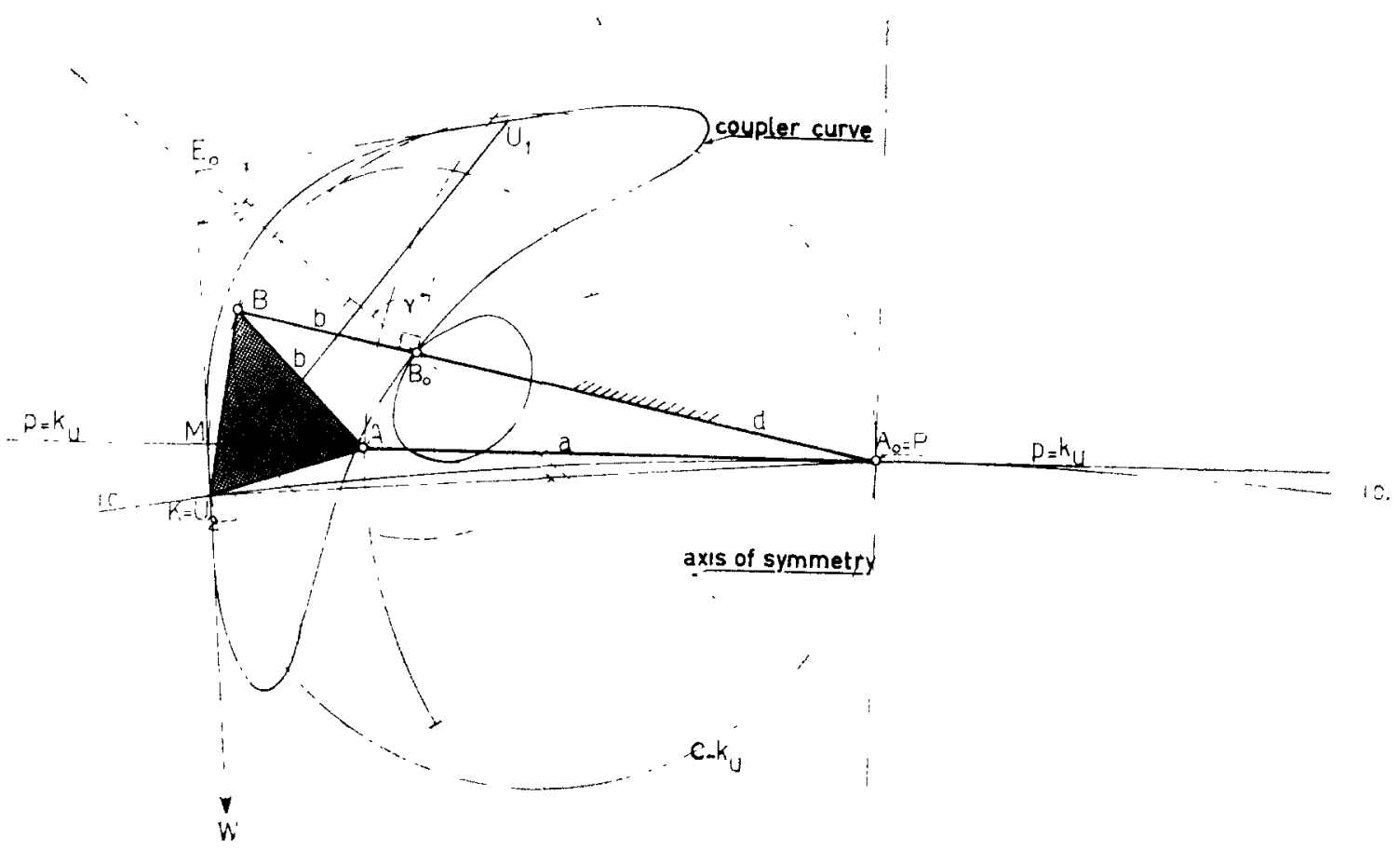

FIG. 20.

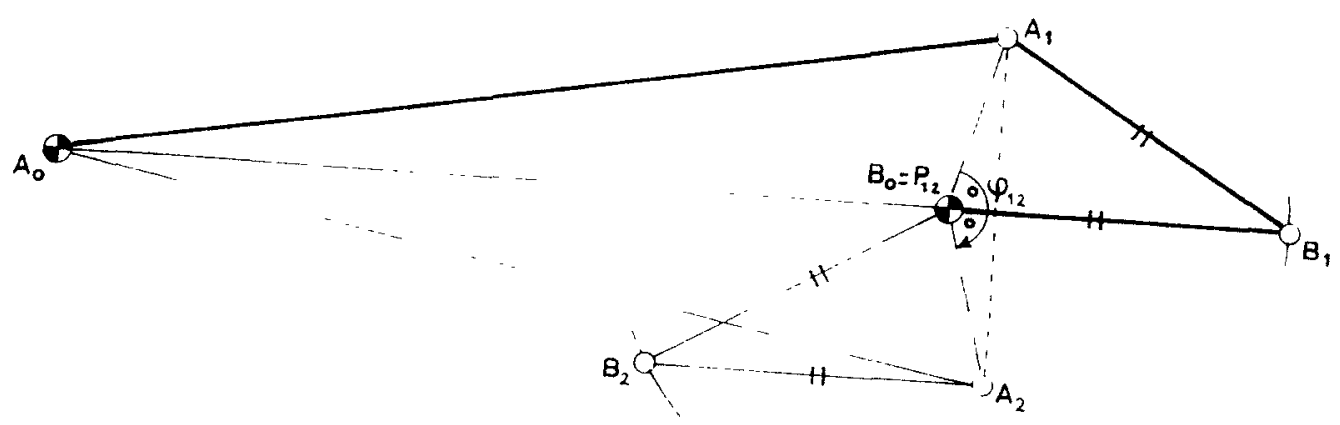

Hik;. 21. 
(b) $A$ on $p$ and $B$ on $c-k_{u}$. In this case $A_{0}=P$ (see Fig. 20). Consequently, points $B, B_{0}$ and $A_{0}$ are in a straight line. In Fig. 21 the coupler corresponding to this position is indicated by $A_{1} B_{1}$. The position in which the coupler point has arrived at the opposite point with respect to the axis of symmetry, corresponds to the position in which turning-point $A$ has arrived at the opposite point $A_{2}$ with respect to the fixed link. It can now be seen from the figure that the two positions of the coupler can be obtained from one another by rotating it about the rotation centre $P_{12}=B_{0}$. Moreover, $\triangle B_{0} A_{1} B_{1} \equiv \triangle B_{0} A_{2} B_{2}$. So $\Varangle B_{2} A_{2} B_{0}=\nless B_{1} B_{0} A_{2}$. This indicates that in the second position the coupler runs parallel to the fixed link, in which case $k_{u}$ and $k_{a}$ are both degenerated: this is the case which we have already discussed in part (b) of section 4.1.1.

(c) Both $A$ and $B$ on $c-k_{u}$ (see Fig. 22). As $\Varangle Q A P=\nless B M P$ and as from the theorem of Bobillier it follows that $\Varangle Q P A=\Varangle B P M, \therefore \triangle P Q A\|\| \triangle P B M$. Hence, $\Varangle P Q A=\Varangle P B M=90^{\circ}$. This means that the collineation axis $P Q \perp A B$.

In Fig. 23 the coupler triangle corresponding to this position is indicated by $A_{1} B_{1} K_{1}\left(P_{1} Q_{1}\right.$ being assumed to be perpendicular to $A_{1} B_{1}$ ). By rotating the crank $A_{0} A$, the coupler triangle arrives at a second position $A_{2} B_{2} K_{2}$ if $A_{2}$ lies opposite to $A_{1}$ with respect to the fixed link. The corresponding coupler points $K_{1}$ and $K_{2}$ are symmetrical with respect to the axis of symmetry of the coupler curve, as can be seen from the fact that the two coupler triangles can reach each other's position by rotating them about the rotation centre $P_{12}=B_{0}$, and from the fact that the axis of symmetry goes through $B_{0}$.

A third position of the coupler triangle, which is in an opposite position to the second with respect to the fixed link, is shown in the same figure. It will be clear that any geometrical property attached to fourbar linkage No. 3 can also be applied to four-bar linkage No. 2.

Now the first and the third four-bar linkages have the crank and the fixed link in common, while the remaining four sides form a rhombus. It will be shown by means of Fig. 24 that the collineation axes $q_{1}$ and $q_{3}$ of the linkages 1 and 3 are parallel, even in any other position of the common crank.

Proof. From $\triangle P_{1} A_{1} B_{1} \| \triangle \triangle A_{3} P_{3} B_{3}$ it follows that ${\overline{P_{1} B_{1}}}_{1} \cdot \bar{P}_{3} B_{3}=\bar{A}_{1} B_{1}, \bar{A}_{3} B_{3}$, so with $\triangle Q_{1} B_{1} B_{0}$ \|\|$B_{0} B_{3} Q_{3}$ we find that

$$
{\overline{Q_{1} B}}_{1} \cdot{\overline{Q_{3} B_{3}}}_{=B_{1} B_{0}} \cdot{\overline{B_{3} B_{0}}}_{=}={\overline{A_{1} B_{1}}}_{1} \cdot{\overline{A_{3} B_{3}}}_{\overline{P_{1} B_{1}}} \cdot{\overline{P_{3} B_{3}}}_{.}
$$

This may be written as

$$
\left({\overline{P_{3}}}_{3} /{\overline{Q_{3} B}}_{3}\right)=\left({\overline{Q_{1} B_{1}}}_{1} /{\overline{P_{1} B_{1}}}\right) \text {, }
$$

so with

we have

$$
\Varangle P_{3} B_{3} Q_{3}=\Varangle Q_{1} B_{1} P_{1}
$$

$$
\triangle P_{3} Q_{3} B_{3}|| \mid \triangle Q_{1} P_{1} B_{1}
$$

Hence $\Varangle B_{3} P_{3} Q_{3}=\Varangle B_{1} Q_{1} P_{1}$, so $q_{1}$ is parallel to $q_{3}$.

Since the collineation axis $P_{1} Q_{1}$ of linkage 1 is perpendicular to the coupler $A_{1} B_{1}$, the collineation axis $P_{3} Q_{3}$ of linkage 3 will be perpendicular to the rocker $B_{3} B_{0}$. The same property holds for linkage 2 .

From the theorem of Bobillier we can then deduce that the crank $A_{2} A_{0}$ is perpendicular to the pole tangent $p_{2}$. So circling point $A_{2}$ lies on $n_{2}$. The equation of $k_{u_{2}}$ shows that if one circling point lies on $n_{2}, n_{2}$ must be a branch of $k_{u 2}$. Another branch of $k_{u_{2}}$ is a circle through $P_{2}$ with its centre on $n_{2}$. So $B_{2}$ lies on $c-k_{u 2}$ as long as position 2 is not a folded position. This leads to the position already investigated under part (c) of section 4.1.1, so that no new viewpoints arise from this consideration.

\subsection{Degeneration of the $k_{a}$ curve, when $l_{\mathrm{o}}^{-1}=0$ (or $R_{\mathrm{o}}=2 R$ )}

In this case only the $k_{a}$ curve degenerates into $p$ and into a circle through $P(0,0)$ and $M(m, 0)$ with its centre on $p$. Ball's point is found at the intersection of the inflection circle with a line through $P$ in the asymptotic direction of $k_{\alpha}$. This line coincides with the pole tangent $p$, so only the pole $P$ can be a Ball's point. Since $P$ is also a cusp, it is not suitable as a coupler point; so in this position there is no solution of the problem.

\subsection{Degeneration of the $k_{u}$ and $k_{a}$ curve, when $P \rightarrow \infty$}

Both the $k_{u}$ and $k_{a}$ curves are cubic. In general both curves pass through the two isotropic points and through one asymptotic point. If the double point of $k_{u}$ and $k_{a}$, i.e. the pole $P$, becomes infinite then the line at infinity will intersect each cubic curve in not less than 5 points. This is only possible when the line at infinity is in itself a branch of $k_{u}$ and $k_{a}$. Then the pole tangent is an asymptotic line of the remaining conic sections, since the two curves touch the pole tangent at $\boldsymbol{P}_{\mathrm{o}}$. Hence the two conic sections are hyperbolas.

The corresponding position of the four-bar linkage will be reached where the crank $A_{0} A$ runs parallel to the rocker $B_{0} B$, as occurs if $P=P_{\infty}$ (see Fig. 25).

\footnotetext{
$\dagger$ Is similar to.
} 
E. A. DIJKSMAN

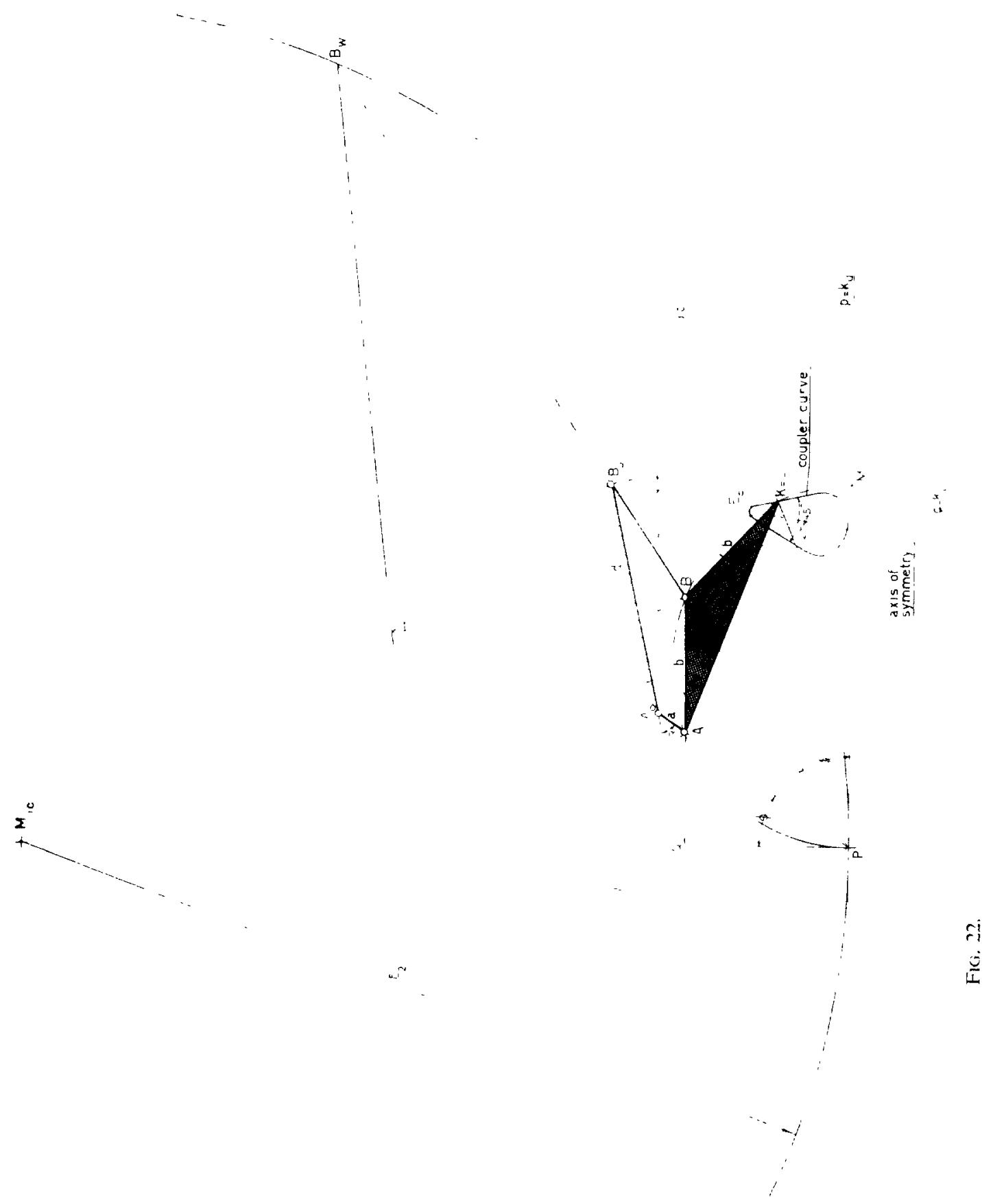




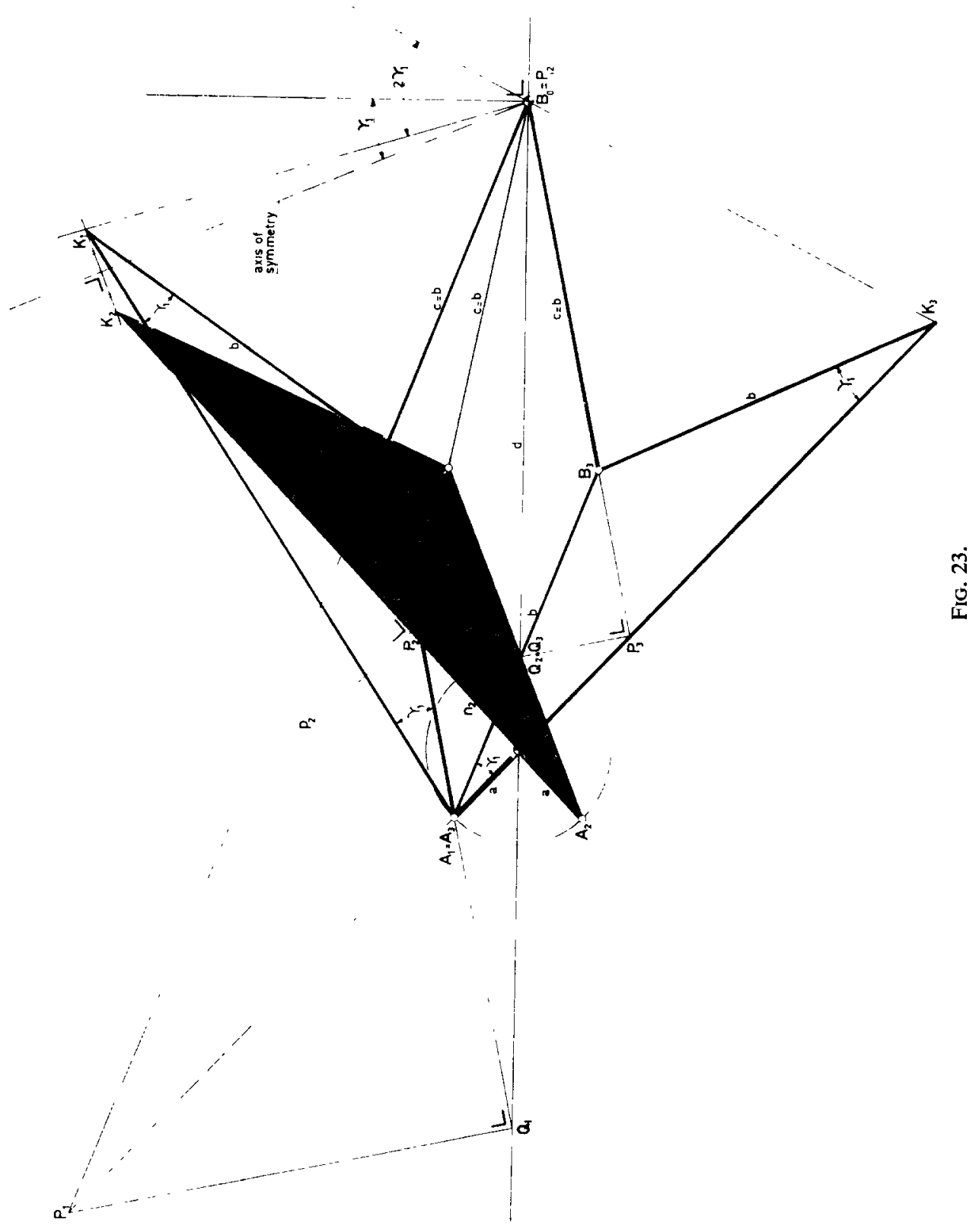




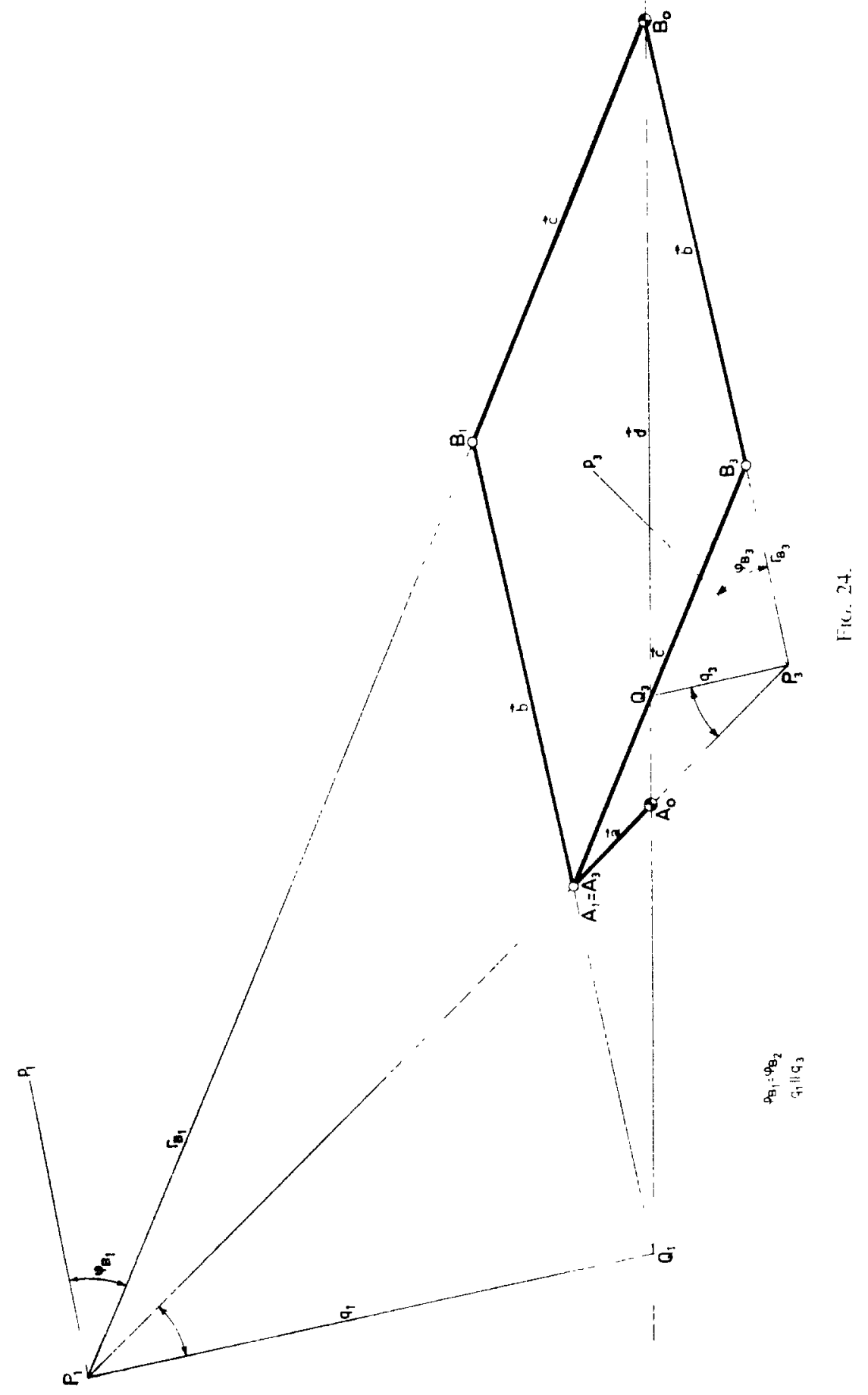




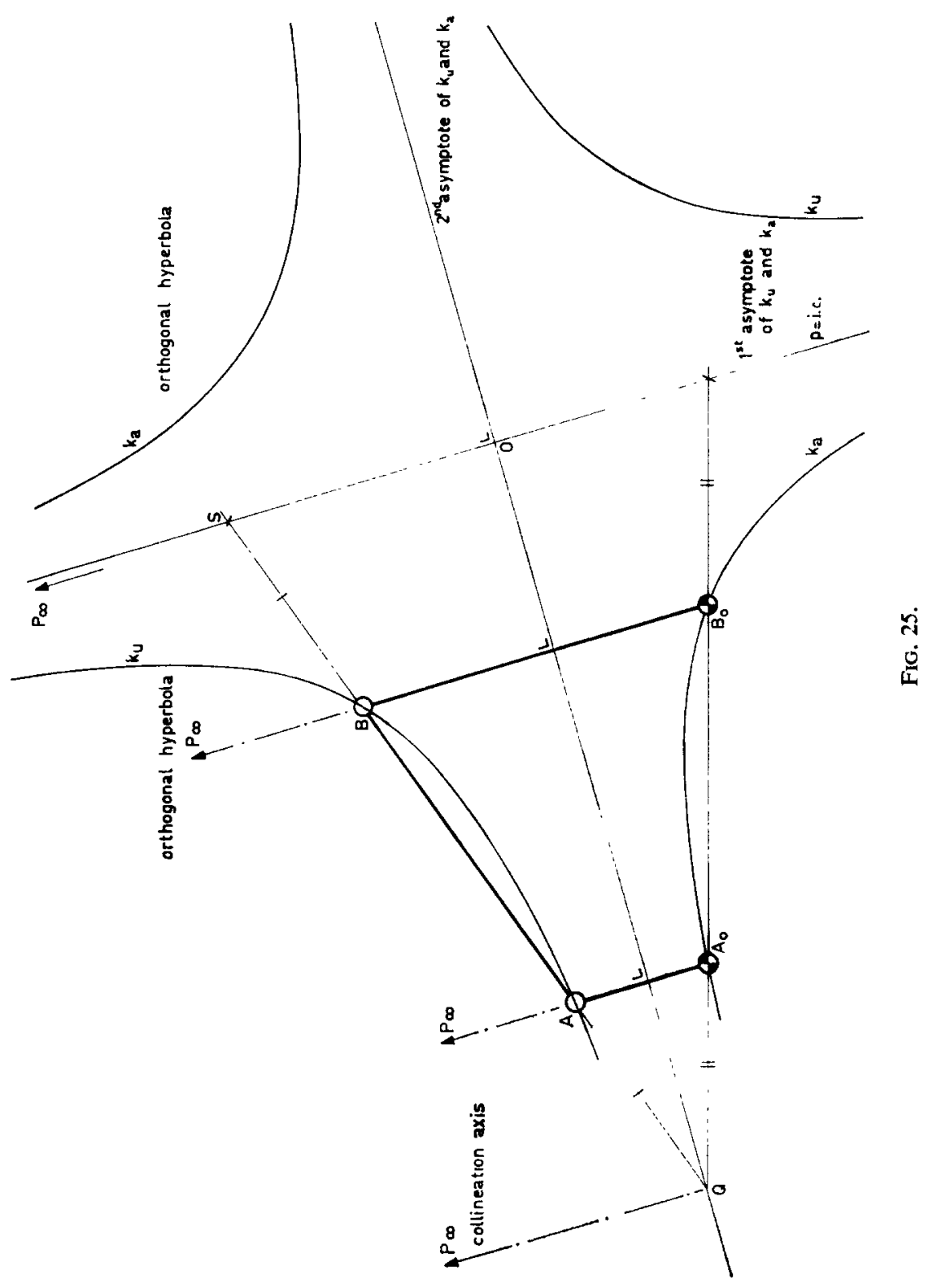




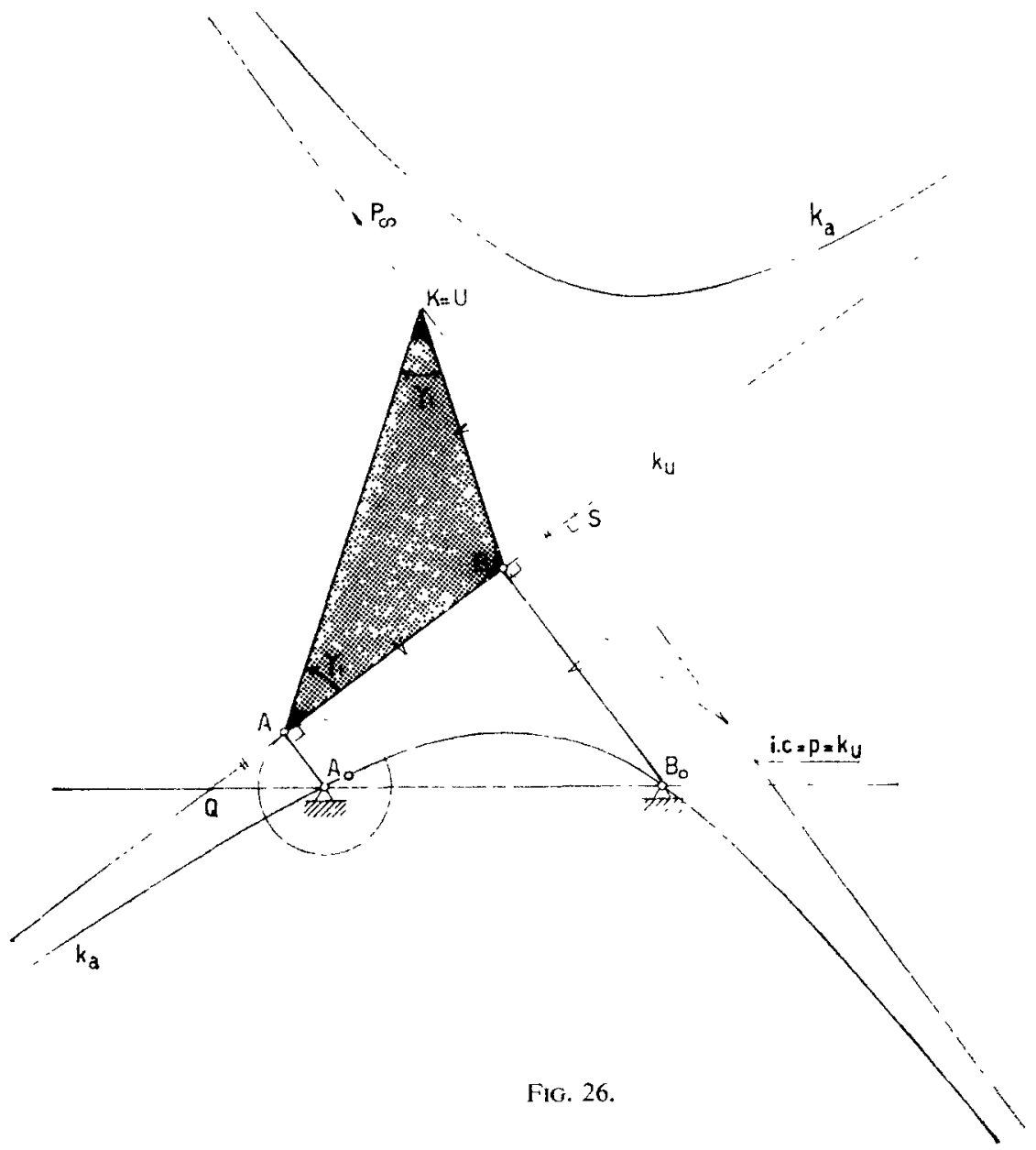

If $Q$ is the collineation point in this position and $S$ the point of intersection of the coupler $A B$ with the pole tangent $p$, it follows from the theorem of Bobillier that $\overline{Q A}=\overline{B S}$.

It is further known that any line intersects a hyperbola in two points; the middle of the distance between these points coincides with the middle of the distance between the intersection points of the line with the asymptotes of the hyperbola. Hence the second asymptote of the hyperbola passes through the collineation point $Q$. Since the second asymptote runs parallel to the pole normal, it is perpendicular to $p$.

A similar reasoning holds for the hyperbola that is a branch of $k_{t}$. Hence both hyperbolas are orthogonal.

The pole tangent $p$ is a branch of the inflection circle, because to each point of $p$ is added a centre of curvature coinciding with $P=P_{\infty}$, as can be seen from the theorem of Hartmann. So in general there is no finite Ball's point that could be a finite intersection of $k_{u}$ and the inflection circle.

This is still true for the case in which the hyperbola that is a branch of $k_{a}$, degenerates into its asymptotes $p$ and $A_{0} B_{0}$. But, if the hyperbola that is a branch of $k_{u}$ degenerates into its asymptotes $p$ and $A B$, then any point of $p$ becomes a Ball's point (see Figs. 26 and 27). In Figs. 26 and 27 the points $A, B_{0}$ and $K$ lie on a chosen circle about $B$, of which $K$ lies also on $p$ since it must be a Ball's point. In Fig. 28 the considered position of the four-bar linkage is indicated by $\left(A_{0} A_{1} B_{1} B_{0}\right)$ and will be referred to as position.1 for short. In position 2 the crankpoint $A$ has reached point $A_{2}$, the opposite of $A_{1}$ with respect to the fixed link $A_{0} B_{0}$. Hence

$$
\triangle A_{2} B_{2} B_{0} \equiv \triangle A_{1} B_{1} B_{0} \text {, so } \Varangle A_{2} B_{2} B_{0}=\Varangle A_{1} B_{1} B_{0}=900 \text {. }
$$

Sinceit follows from position 1 that $d^{2}=b^{2}+(b-a)^{2}, A_{2} A_{0}$ coincides with $A_{2} B_{2}$, and therefore position 2 corresponds with the cardan position; this case was discussed in section 4.2 . We conclude that starting from position 1, we can only expect to find solutions such as have already been calculated with the cardan position as the starting position. 

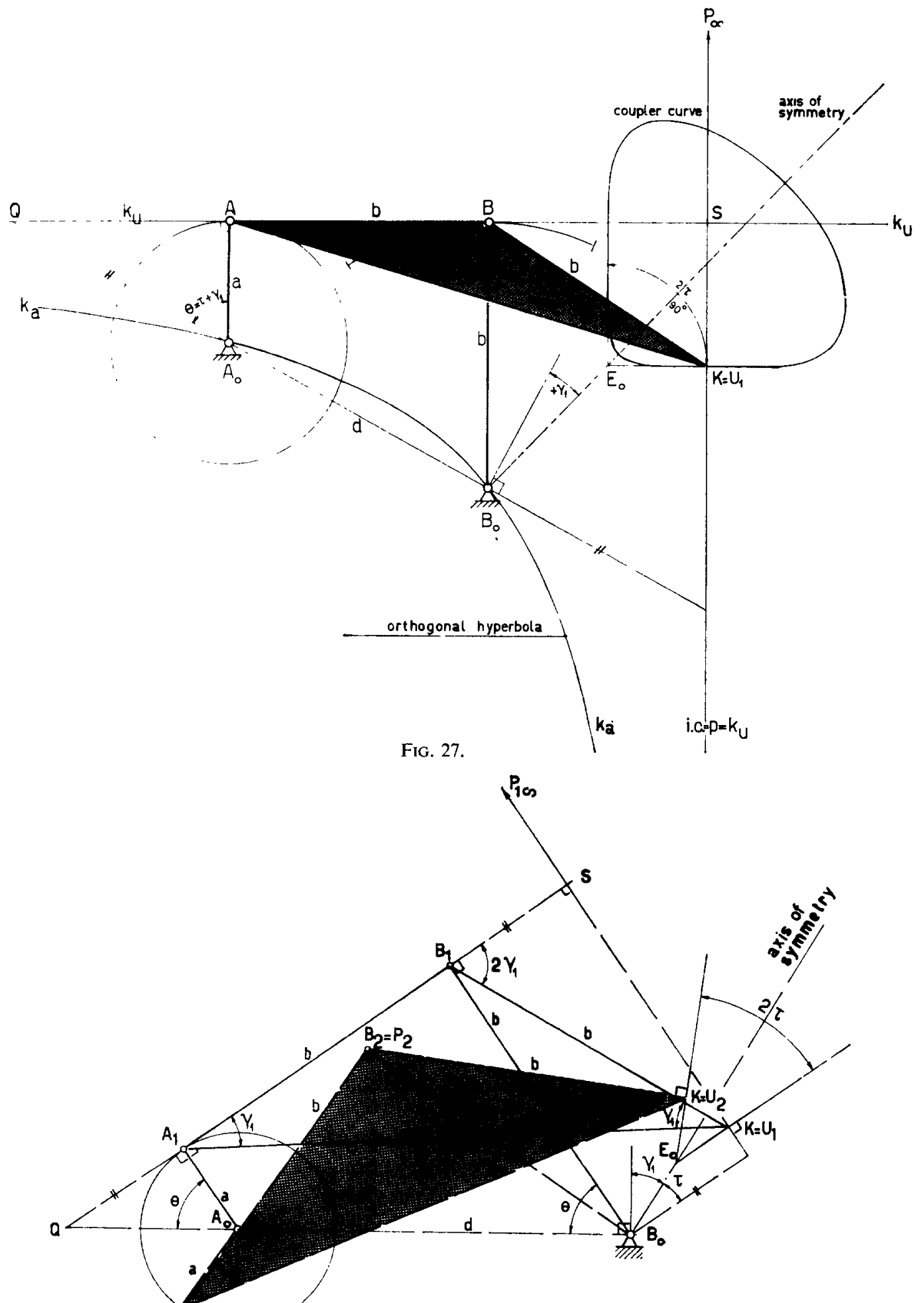

FIG. 28. 


\section{INVESTIGATION OF THE MAIN PROBLEM}

In what precedes, all possibilities of degeneration of $k_{u}$ have been investigated in connection with the production of $V$-shaped, symmetrical coupler curves by four-bar linkages.

When $k_{u}$ is not of degenerate form, we get one more degree of freedom in design. This additional degree of freedom may then be used for different purposes. It allows us, for example, to choose the coupler point $K$ on the produced part of the coupler for any given angle $2 \tau$. (The choice corresponds to the condition $\gamma_{1}=0^{\circ}$.) The corresponding dimensions are calculated and given in Table 3. Besides, Figs. 29, 30, 31 and 32 show how the Geneva wheel moves with respect to time for $2 \tau=45^{\circ}, 60^{\circ}, 90^{\circ}$ and $120^{\circ}$.

TABle 3. Solutions With $B$ INSIDE THL INFLECTION CIRCLE AND $\gamma_{1}=0$

\begin{tabular}{|c|c|c|c|c|c|c|}
\hline $2 \tau$ & $120^{\prime \prime}$ & $90^{3}$ & 72 & 60 & $45^{\prime \prime}$ & $30^{\circ}$ \\
\hline$a / b$ & 0.4988 & $0 \cdot 4630$ & 0.4187 & 0.3787 & 0.3152 & 0.2339 \\
\hline$d / b$ & $1 \cdot 1514$ & $1 \cdot 3018$ & $1 \cdot 4015$ & 14749 & 1.5773 & 1.6952 \\
\hline$\mu_{1}$ & $68 \cdot 80^{\prime}$ & $56 \cdot 14$ & 4894 & $4410^{-}$ & $37 \cdot 73$ & $30 \cdot 58$ \\
\hline$\mu_{2}$ & $38.09^{\prime \prime}$ & $49 \cdot 59$ & 58.86 & $66 \cdot 47$ & 78.25 & 93.87 \\
\hline$\overline{B_{0} E_{0}} / b$ & 1.0688 & 0.8715 & 0.7597 & 06846 & () 5861 & 0.4756 \\
\hline${\overline{E_{0} U_{1}}}_{1} b$ & 0.4870 & 0.4257 & 0.3856 & 0.3564 & 0.3154 & 0.2649 \\
\hline 0 & 62.78 & $58 \cdot 75^{\prime}$ & 55.77 & $53 \cdot 62^{\prime}$ & $50 \cdot 82$ & 4790 \\
\hline$h / b$ & 0.7607 & 0.8746 & 0.9133 & 0.9219 & 0.9046 & 0.8379 \\
\hline$\left(\rho T_{1}\right) / b$ & 0.2877 & $0 \cdot 1058$ & 0.0546 & 0.0330 & 0.0154 & 0.0055 \\
\hline$\left(\rho T_{2}\right) / b$ & $1 \cdot 4825$ & $1 \cdot 1346$ & 0.8432 & 0.6260 & 0.3598 & 0.1468 \\
\hline$\varphi_{B}$ & $1.42^{\prime \prime}$ & $7 \cdot 51$ & 11.05 & $13 \cdot 35$ & $16 \cdot 17$ & 18.90 \\
\hline$\varepsilon_{m}$ & 7756.07 & $63 \cdot 98$ & $20 \cdot 87$ & $11 \cdot 81$ & $6 \cdot 45$ & 377 \\
\hline
\end{tabular}

It is also possible to pursue an optimal $\mu_{\min }$ for each angle $2 \tau$. The dimensions complying with this optimum are represented in Table 4 . With the aid of the data taken from this table, drawings have been made of the driving mechanisms for $n=3,4,5,6,8$ and 12 stations (see Figs. 34 to 39). Moreover, graphs of the resultant motion of the Geneva wheel are shown in Figs. 40 to 43 for $n=8,6,4$ and 3 respectively.

The calculations behind the general case are based on the following construction (see Fig. 44):

(a) Draw the pole tangent $p$, the pole normal $n$, and the inflection circle. 


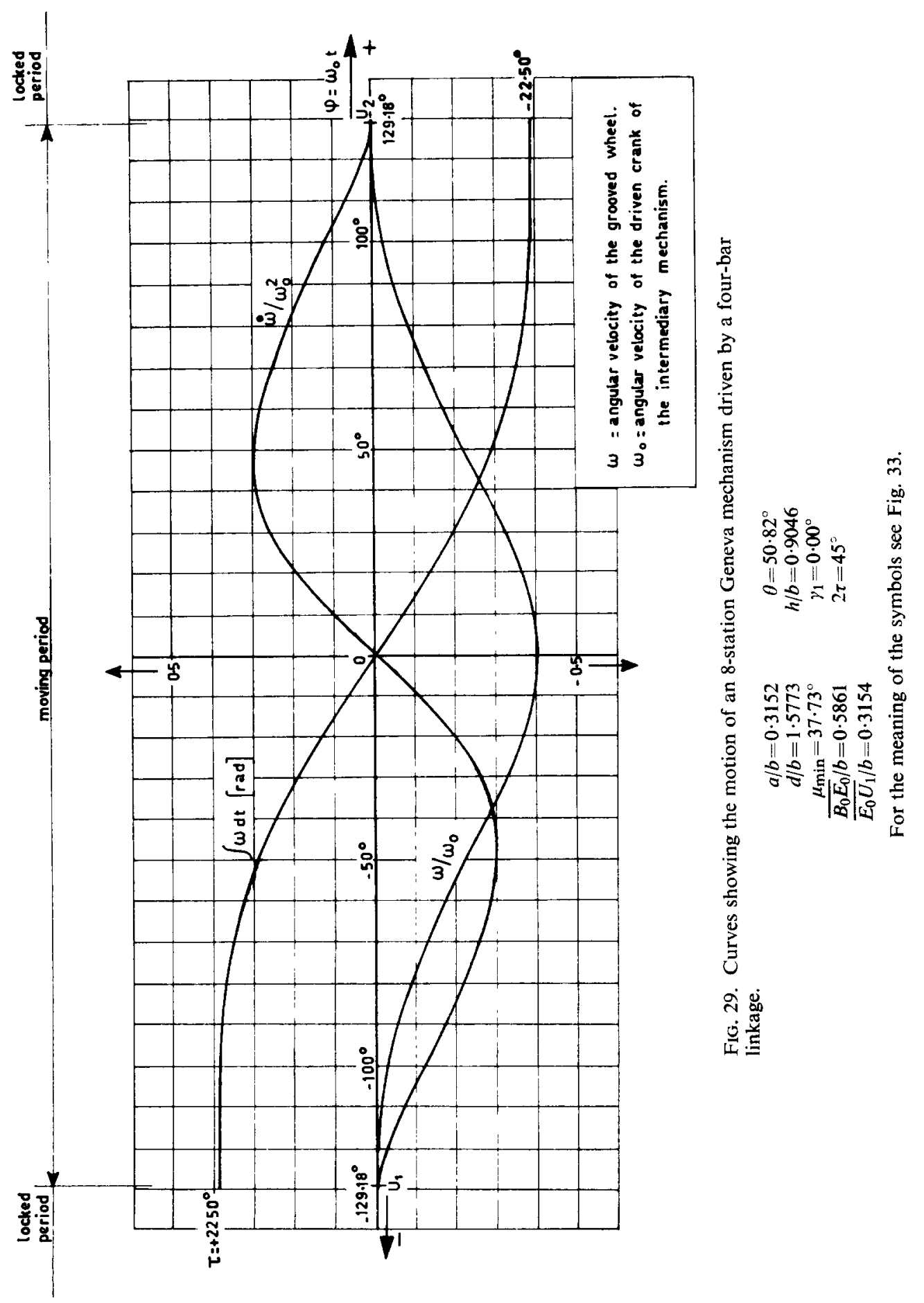




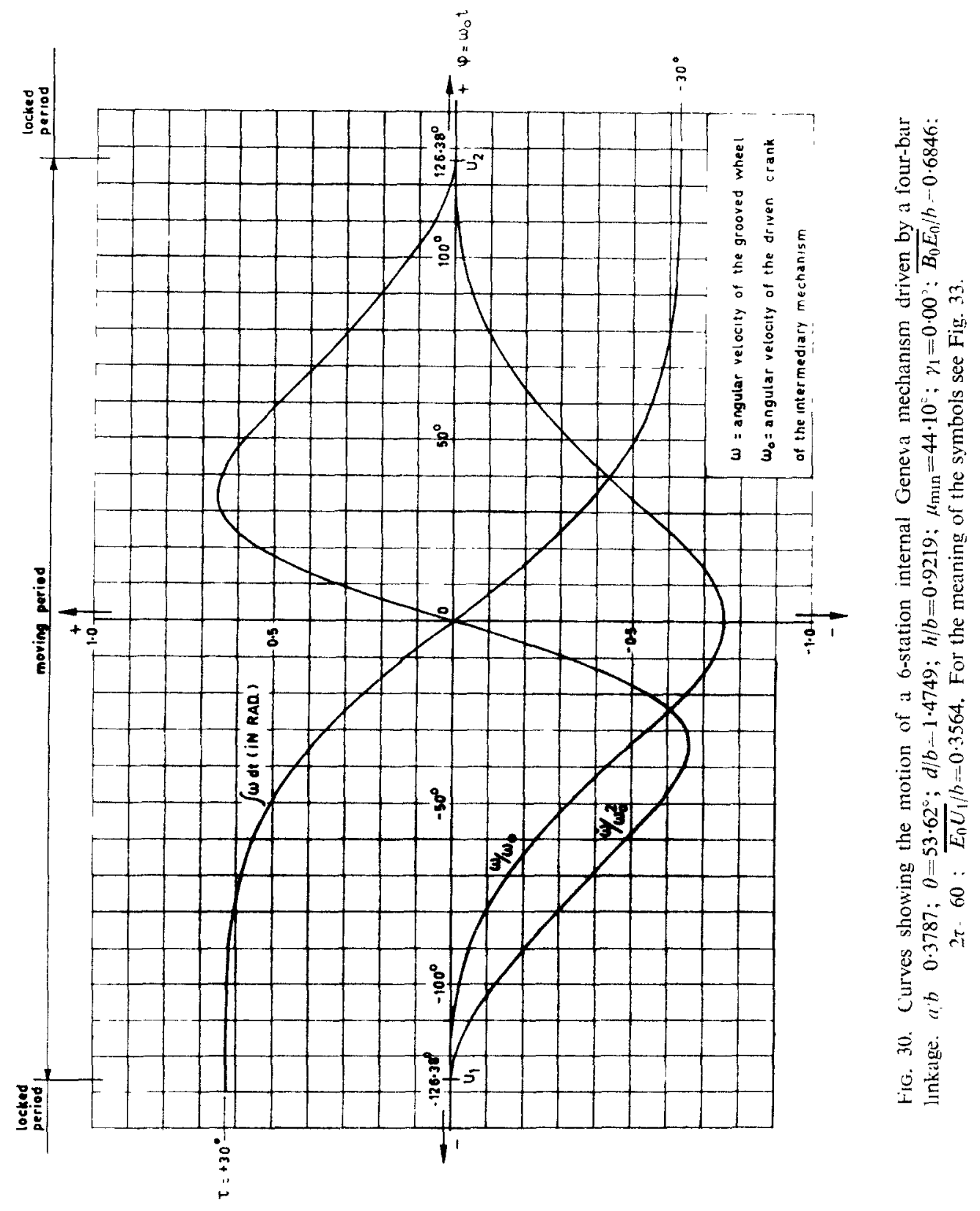




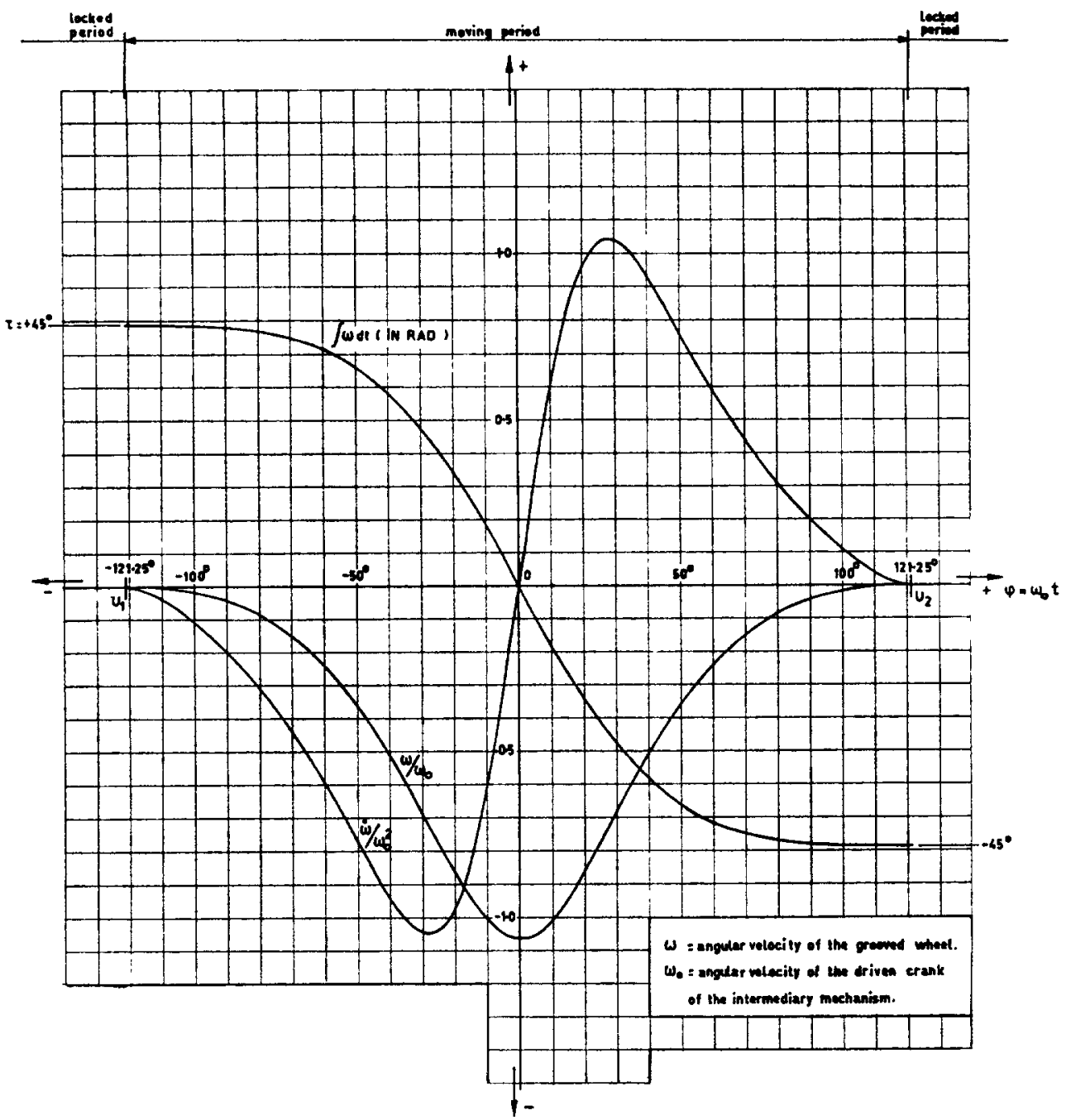

Fig. 31. Curves showing the motion of a 4-station internal Geneva mechanism driven by a four-bar linkage.

$$
\begin{array}{rlrl}
a / b & =0.4630 & \theta & =58.75^{\circ} \\
d / b & =1.3018 & h / b & =0.8746 \\
\mu_{\min } & =49.59^{\circ} & \gamma_{1} & =0.00^{\circ} \\
\overline{B_{0} E_{0}} / b & =0.8715 & 2 \tau & =90^{\circ} \\
\overline{E_{0} U_{1}} / b & =0.4257 & &
\end{array}
$$

For the meaning of the symbols see Fig. 33. 


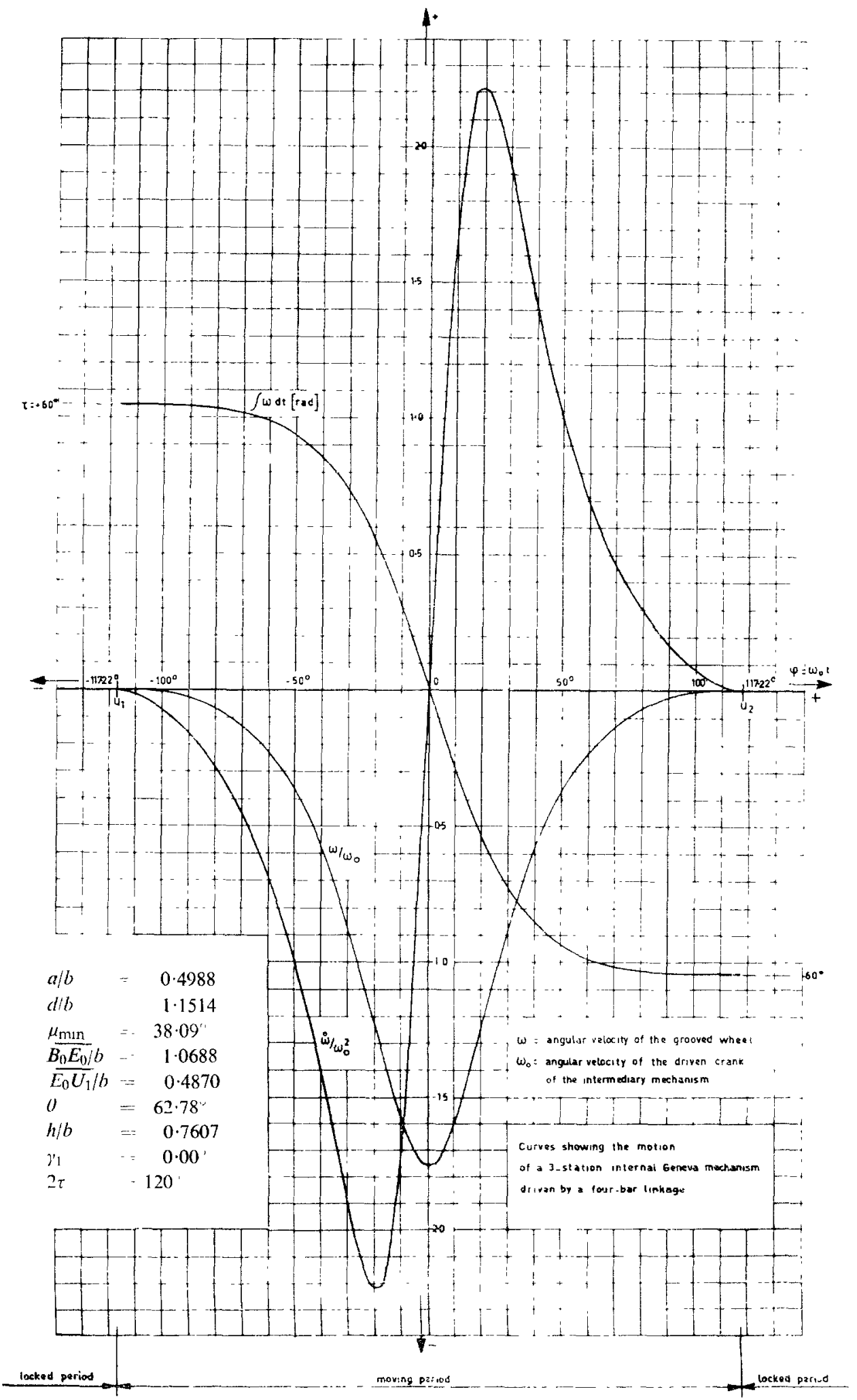

Fig. 32. For the meaning of the symbols see Fig. 33 


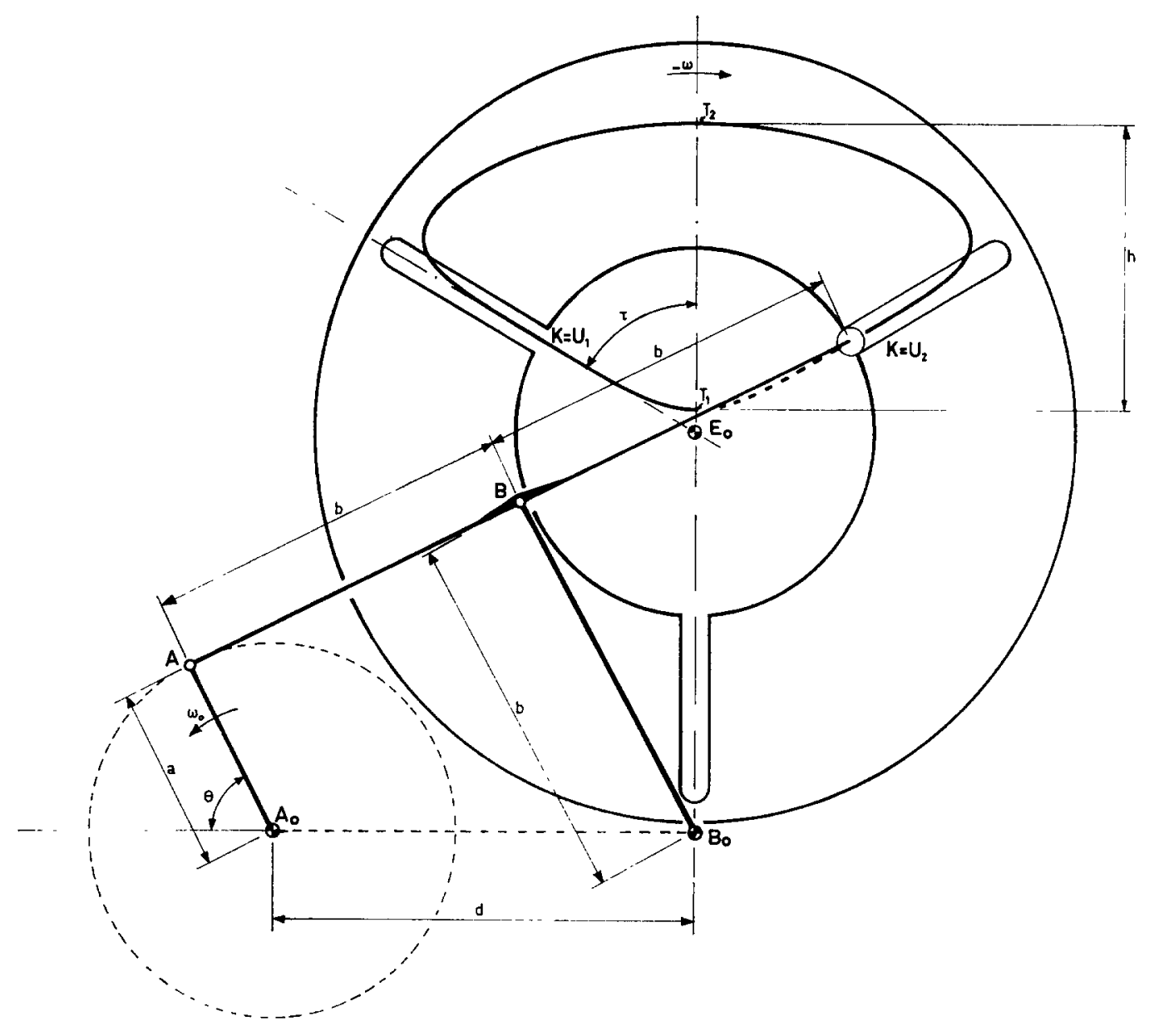

Fig. 33. 


\begin{tabular}{|c|c|c|c|c|c|c|c|c|c|c|c|c|}
\hline in & $\frac{\tilde{\sigma}}{\dot{0}}$ & 总 & $\frac{\dot{m}}{\dot{q}}$ & $\frac{\partial}{\dot{j}}$ & $\stackrel{g}{g}$ & $\begin{array}{l}\stackrel{P}{\leftrightarrow} \\
\stackrel{N}{0} \\
\dot{0}\end{array}$ & $\begin{array}{l}\infty \\
\infty \\
\dot{0} \\
\dot{8}\end{array}$ & $\frac{\tilde{\sigma}}{\sigma}$ & $\begin{array}{l}8 \\
\stackrel{8}{0} \\
\dot{m}\end{array}$ & $\frac{\stackrel{9}{3}}{\dot{\gamma}}$ & $\overline{\dot{m}}$ & $\frac{q}{\dot{m}}$ \\
\hline in & $\stackrel{\infty}{\stackrel{8}{0}}$ & 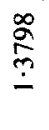 & $\begin{array}{l}\stackrel{0}{\sim} \\
\dot{\sigma}\end{array}$ & 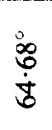 & $\begin{array}{l}\stackrel{N}{\infty} \\
\infty \\
\infty\end{array}$ & $\begin{array}{l}\stackrel{\sim}{f} \\
\stackrel{m}{0}\end{array}$ & $\begin{array}{l}\stackrel{\circ}{\alpha} \\
\dot{8}\end{array}$ & $\begin{array}{l}\frac{\infty}{0} \\
\stackrel{0}{0} \\
\dot{0}\end{array}$ & $\frac{\stackrel{8}{7}}{\dot{7}}$ & $\frac{P}{\dot{P}}$ & $\frac{\stackrel{2}{\sigma}}{\dot{b}}$ & $\hat{m}$ \\
\hline$\stackrel{8}{n}$ & $\begin{array}{l}\stackrel{1}{\circ} \\
\stackrel{\overbrace{}}{\circ} \\
\dot{0}\end{array}$ & $\begin{array}{l}\approx \\
\stackrel{n}{=}\end{array}$ & $\begin{array}{l}\stackrel{\infty}{\infty} \\
\stackrel{1}{m}\end{array}$ & $\frac{i}{\dot{n}}$ & 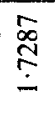 & $\mid \begin{array}{c}\tilde{N} \\
\dot{y} \\
\dot{0}\end{array}$ & ${ }_{0}^{\infty}+\infty$ & $\begin{array}{l}\hat{\oplus} \\
\stackrel{\hat{\theta}}{0}\end{array}$ & 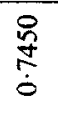 & $\frac{\vec{\infty}}{\vec{n}}$ & ஸे & $\stackrel{\infty}{\doteq}$ \\
\hline in & $\stackrel{a}{7}$ & $\begin{array}{l}\stackrel{m}{g} \\
\stackrel{m}{-}\end{array}$ & $\begin{array}{l}\stackrel{\rho}{\mathscr{H}} \\
\dot{\sim}\end{array}$ & $\stackrel{n}{\hat{n}}$ & $\begin{array}{l}\stackrel{8}{\circ} \\
\stackrel{n}{2}\end{array}$ & $\begin{array}{l}\text { 苛 } \\
\text { 号 } \\
0\end{array}$ & 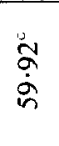 & 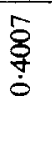 & $\begin{array}{l}\bar{J} \\
\stackrel{5}{5} \\
\dot{0}\end{array}$ & $\frac{\frac{m}{d}}{\frac{9}{-}}$ & $\stackrel{\ddot{r}}{\grave{0}}$ & $\frac{a}{\stackrel{a}{n}}$ \\
\hline 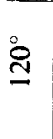 & 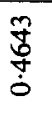 & $\stackrel{n}{n}$ & $\begin{array}{l}\stackrel{\sim}{N} \\
\dot{\sigma}\end{array}$ & $\frac{\stackrel{0}{8}}{\frac{1}{n}}$ & $\stackrel{\bar{n}}{\stackrel{n}{-}}$ & 古 & $\stackrel{\stackrel{m}{\hat{m}}}{\dot{\hat{n}}}$ & $\underset{\substack{n \\
0 \\
0}}{2}$ & $\begin{array}{l}\overrightarrow{\tilde{D}} \\
\stackrel{\sim}{\sim} \\
\dot{0}\end{array}$ & 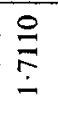 & $\begin{array}{l}\dot{6} \\
\dot{0}\end{array}$ & 产 \\
\hline$\stackrel{0}{0}$ & 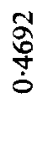 & $\stackrel{g}{\stackrel{g}{\leftrightarrows}}$ & 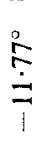 & 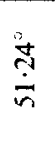 & $\frac{8}{\stackrel{8}{Z}}$ & $\begin{array}{l}\overline{5} \\
\stackrel{5}{+} \\
\dot{0}\end{array}$ & $\underset{\substack{\infty \\
\infty \\
\infty}}{\stackrel{0}{n}}$ & 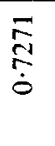 & $\frac{\infty}{\stackrel{0}{9}}$ & 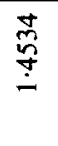 & $\begin{array}{l}0^{\circ} \\
\dot{0} \\
\dot{0}\end{array}$ & $\begin{array}{l}\dot{n} \\
\tilde{n}\end{array}$ \\
\hline \& & 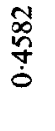 & 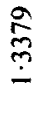 & 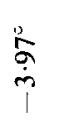 & $\frac{\infty}{\dot{\sim}}$ & $\frac{0}{a}$ & ڤ్ & $\frac{\infty}{\infty}$ & 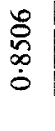 & $\frac{\infty}{5}$ & $\frac{a}{2}$ & $\begin{array}{l}\tilde{\sigma} \\
\alpha\end{array}$ & $\begin{array}{l}\stackrel{R}{n} \\
\dot{n}\end{array}$ \\
\hline$\stackrel{3}{N}$ & $\begin{array}{l}\stackrel{0}{7} \\
\stackrel{y}{0} \\
\dot{0}\end{array}$ & $\stackrel{\text { S }}{\stackrel{\Xi}{=}}$ & $\begin{array}{l}i o \\
\dot{n} \\
+\end{array}$ & $\stackrel{m}{\dot{m}}$ & $\begin{array}{l}\frac{P}{5} \\
\dot{6} \\
\dot{0}\end{array}$ & $\frac{g}{\tilde{g}}$ & 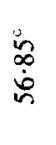 & $\begin{array}{c}\bar{\infty} \\
\stackrel{d}{0} \\
\dot{0}\end{array}$ & $\begin{array}{l}\overrightarrow{\hat{N}} \\
\dot{\delta} \\
\dot{0}\end{array}$ & $\begin{array}{l}\bar{\infty} \\
\stackrel{\sigma}{0}\end{array}$ & $\begin{array}{l}\stackrel{i}{\infty} \\
\dot{\infty} \\
i\end{array}$ & 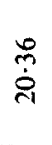 \\
\hline 8 & 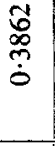 & $\begin{array}{l}\stackrel{8}{8} \\
\stackrel{D}{-}\end{array}$ & 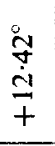 & $\begin{array}{l}\stackrel{0}{0} \\
\infty \\
\infty \\
n\end{array}$ & $\underset{\tilde{n}}{\stackrel{n}{5}}$ & $\begin{array}{c}\infty \\
\tilde{N} \\
\tilde{0} \\
\dot{0}\end{array}$ & 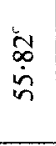 & $\begin{array}{l}\mathscr{\delta} \\
\text { ô } \\
\dot{0}\end{array}$ & $\begin{array}{l}\tilde{D} \\
\tilde{O} \\
\dot{0}\end{array}$ & 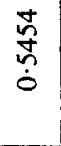 & 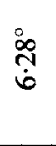 & 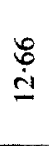 \\
\hline 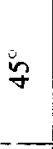 & $\underset{\overbrace =}{\stackrel{\overbrace{}}{0}}$ & 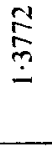 & $\begin{array}{l}\infty \\
\infty \\
\dot{\hat{H}} \\
+ \\
\end{array}$ & $\stackrel{i}{\hat{b}}$ & 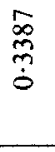 & $\frac{\tilde{n}}{\tilde{\sigma}}$ & $\begin{array}{l}\stackrel{\circ}{\dot{S}} \\
\dot{+}\end{array}$ & 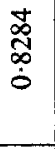 & $\stackrel{\bar{\exists}}{\stackrel{\bar{O}}{\dot{\theta}}}$ & $\begin{array}{l}8 \\
\stackrel{8}{0} \\
\dot{1} \\
\dot{0}\end{array}$ & 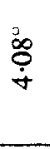 & $\begin{array}{l}\infty \\
\infty \\
\infty\end{array}$ \\
\hline ల్ల & $\begin{array}{c}\hat{\tilde{N}} \\
\stackrel{0}{0}\end{array}$ & 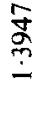 & $\begin{array}{l}\hat{0} \\
\stackrel{\hat{i}}{+} \\
+\end{array}$ & $\begin{array}{l}\hat{\dot{g}} \\
\dot{\hat{g}}\end{array}$ & $\frac{a}{a}$ & $\begin{array}{l}\infty \\
\infty \\
\frac{0}{2} \\
\dot{0}\end{array}$ & $\frac{8}{\square}$ & 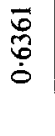 & $\begin{array}{l}\stackrel{9}{0} \\
\stackrel{8}{0}\end{array}$ & $\begin{array}{l}\infty \\
2 \\
0 \\
\stackrel{0}{0} \\
\end{array}$ & $\stackrel{\infty}{\stackrel{\infty}{\leftrightarrows}}$ & $\underset{\dot{\infty}}{\infty}$ \\
\hline$\approx$ & $\stackrel{\overrightarrow{3}}{\dot{0}}$ & 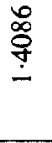 & $\begin{array}{l}\stackrel{\circ}{\hat{\sigma}} \\
\stackrel{m}{+} \\
+\end{array}$ & $\begin{array}{l}\stackrel{+}{\infty} \\
\stackrel{2}{r}\end{array}$ & 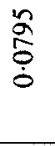 & $\stackrel{+}{\tilde{S}}$ & $\begin{array}{l}\infty \\
⿱ \infty \\
\dot{q} \\
\dot{q}\end{array}$ & $\begin{array}{l}5 \\
\tilde{8} \\
\tilde{0}\end{array}$ & $\begin{array}{l}0 \\
\tilde{\delta}\end{array}$ & $\stackrel{\text { ப }}{\stackrel{0}{0}}$ & $\begin{array}{l}P \\
0 \\
0\end{array}$ & $\overline{0}$ \\
\hline$\stackrel{N}{N}$ & $\pi$ & $\mathbb{s}$ & $\Sigma$ & 丞 & 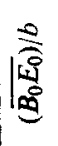 & 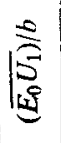 & 0 & 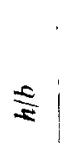 & $\stackrel{\varrho}{\stackrel{E}{E}}$ & $\frac{\overline{\hat{N}}}{\mathrm{~S}}$ & $\stackrel{5}{Q}$ & E \\
\hline
\end{tabular}




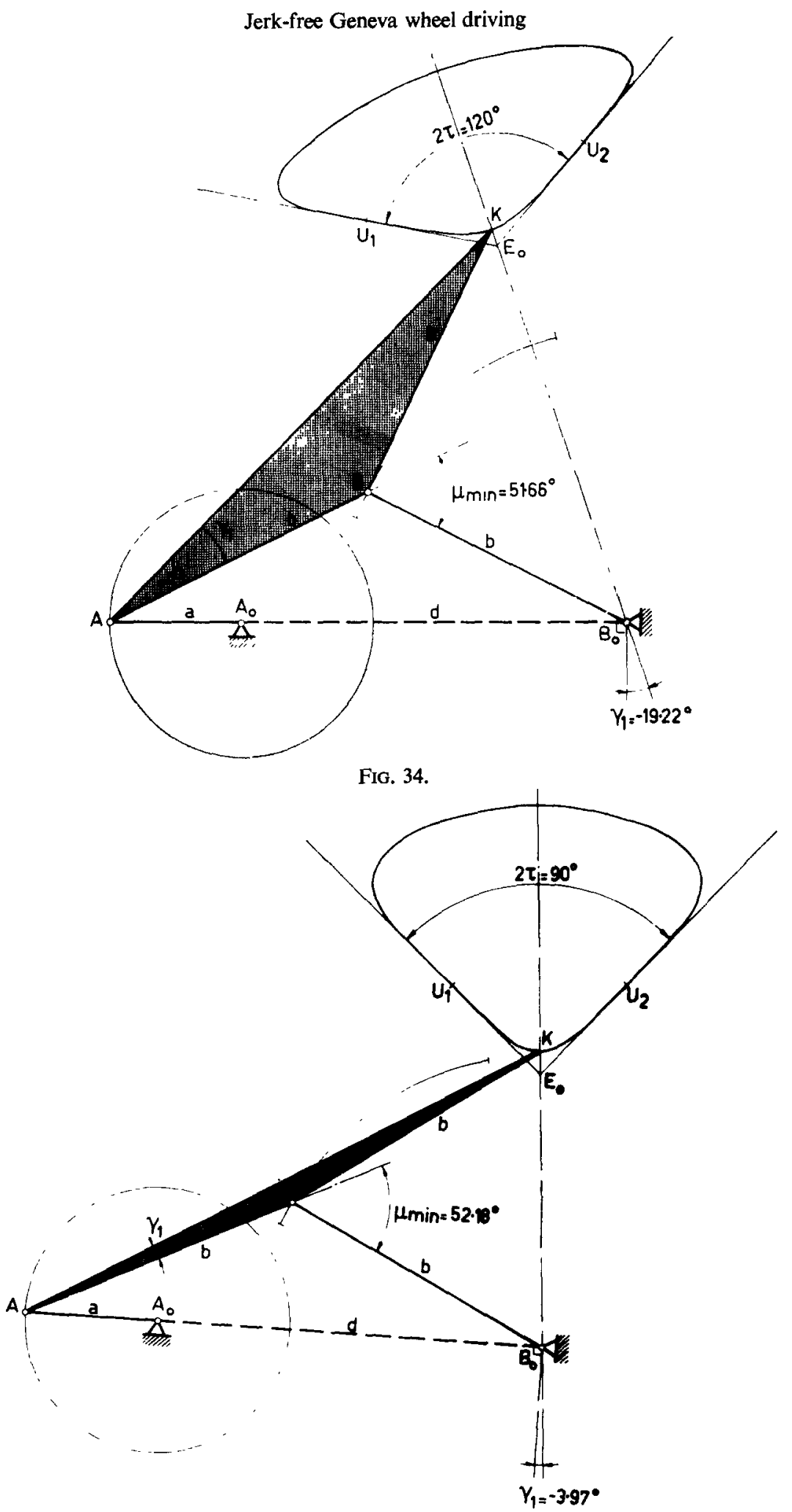

FIG. 35 . 


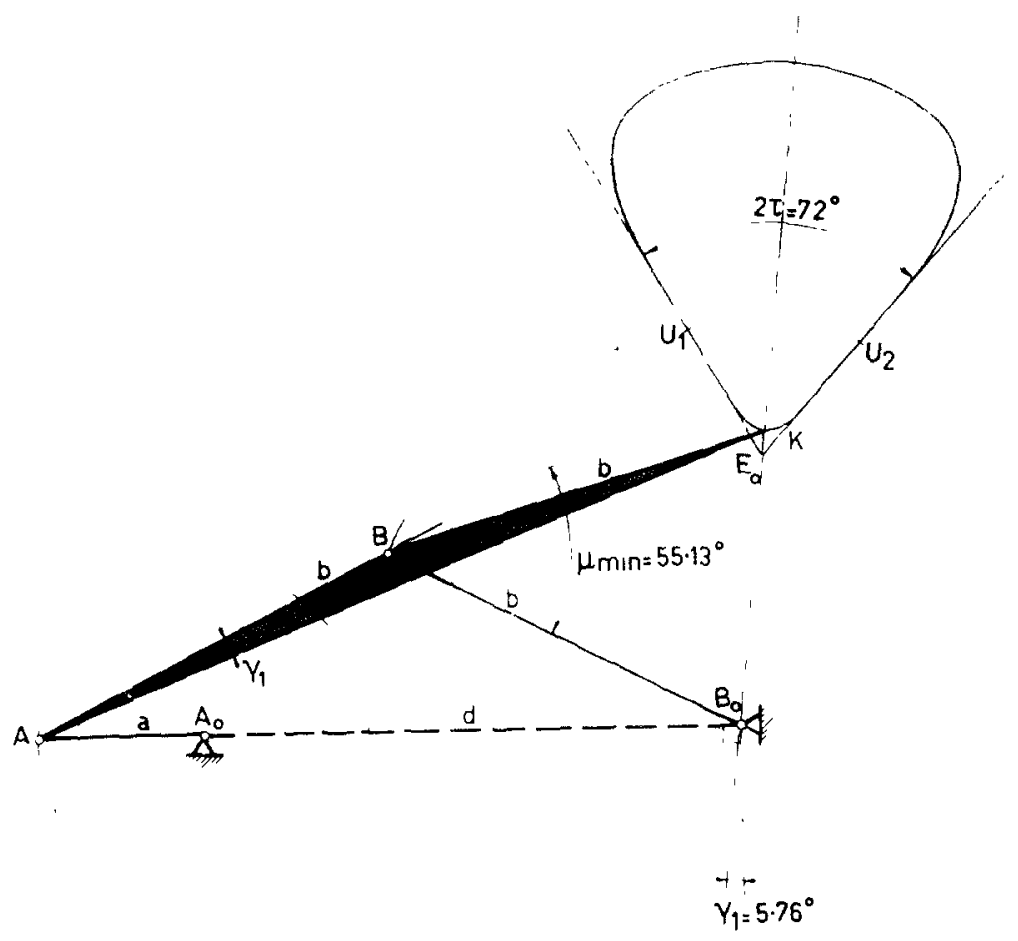

Fici. 36.

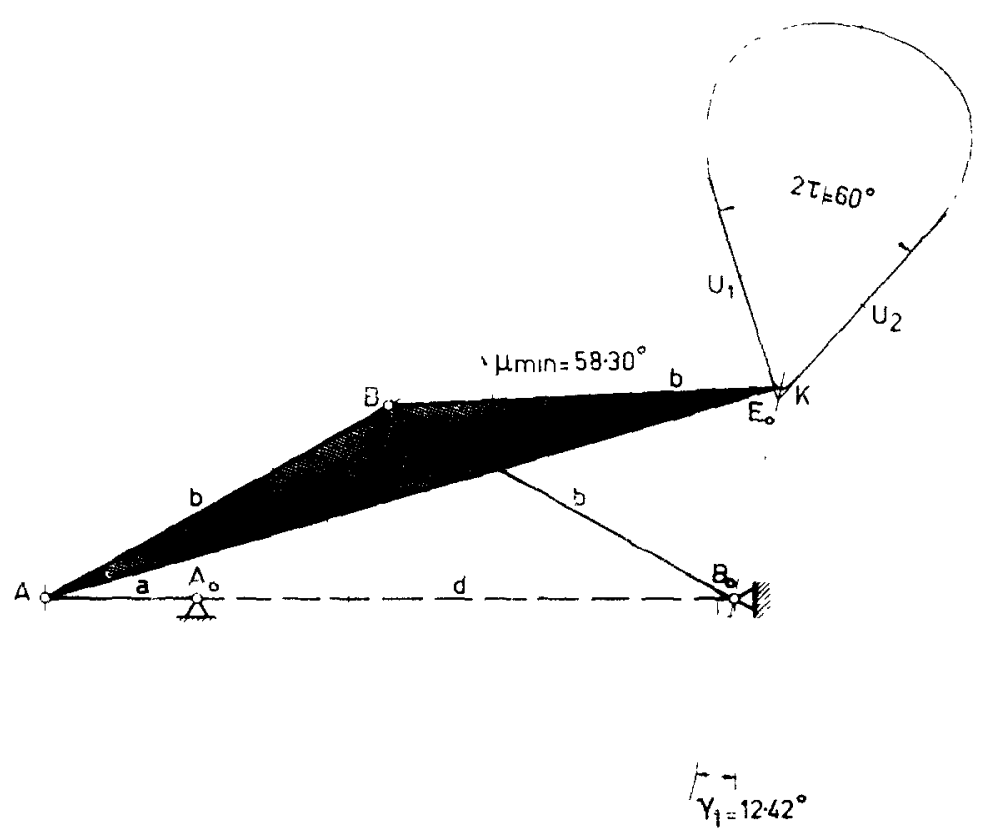

FIC. 37. 


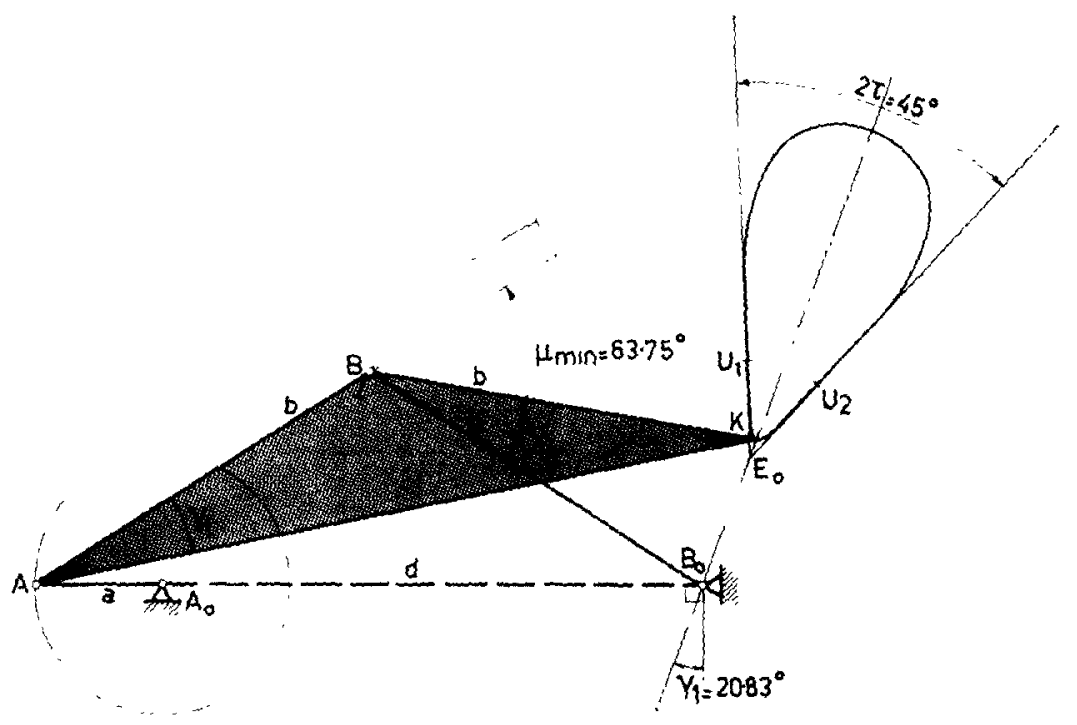

FIG. 38.

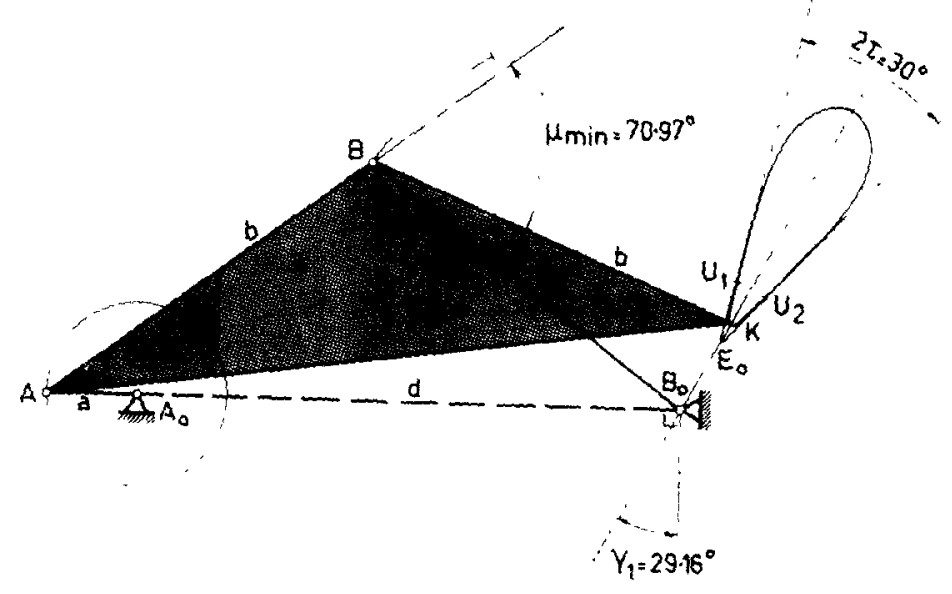

Fic. 39. 


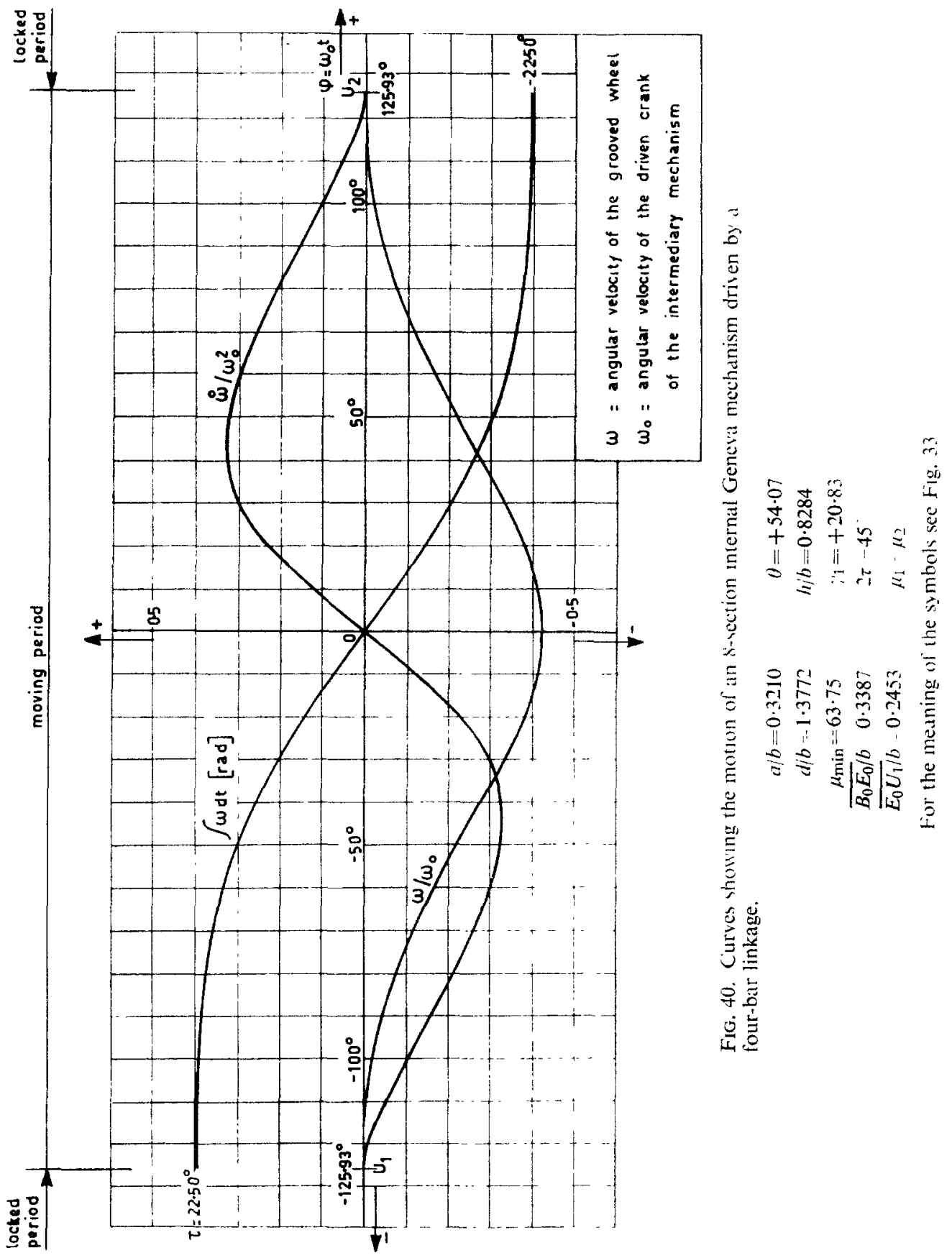




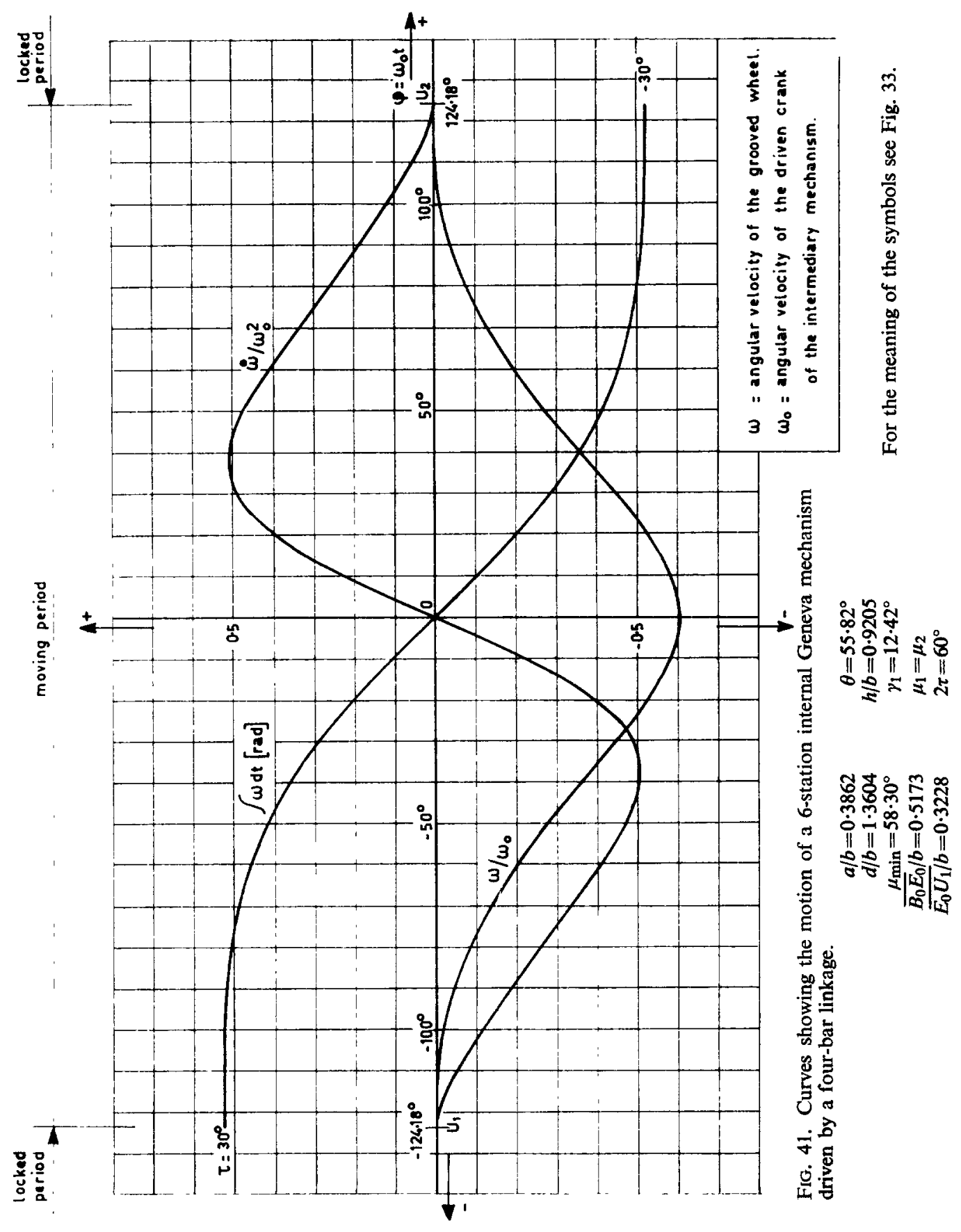




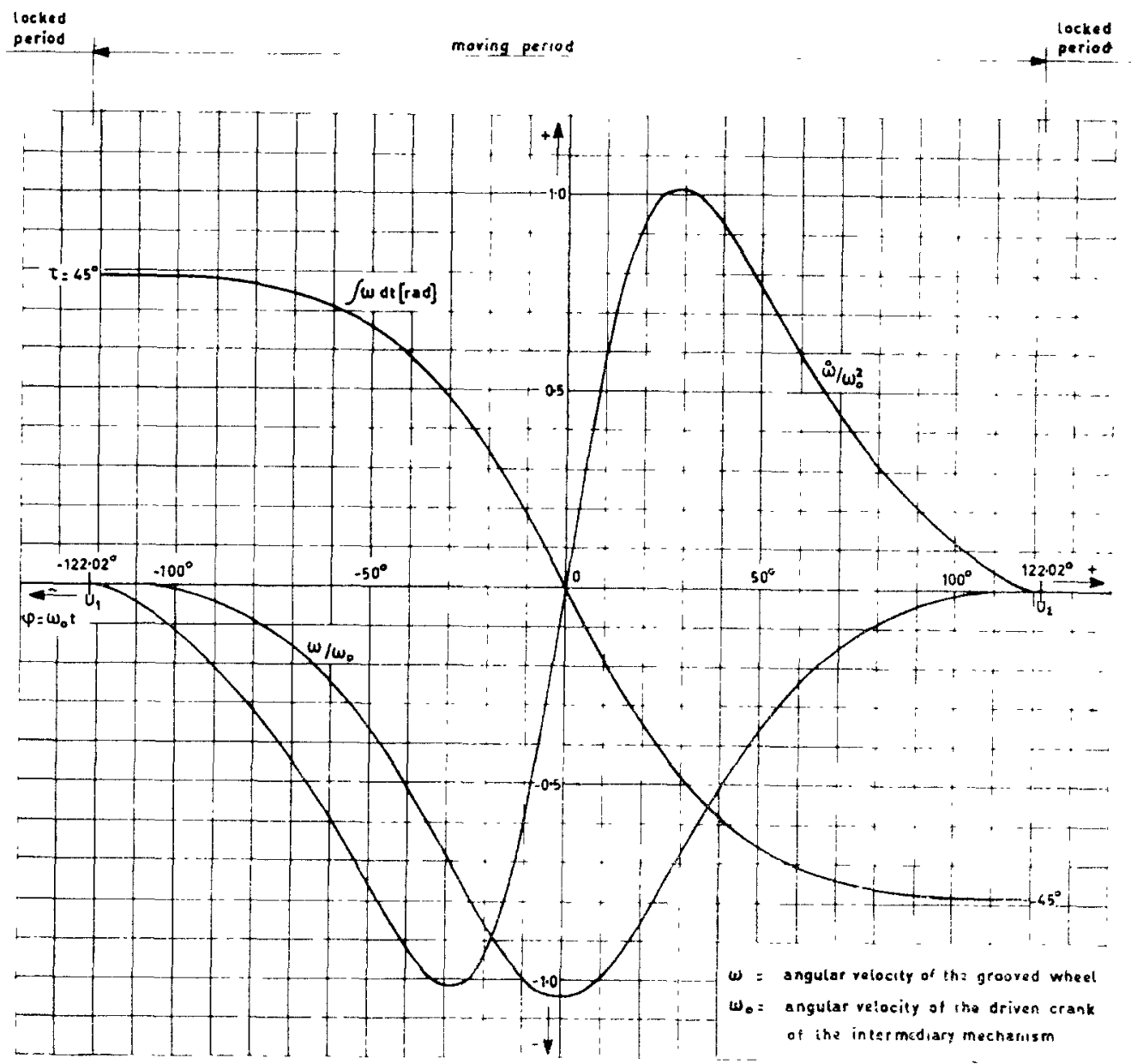

Fig. 42, Curves showing the motion of a t-station mternal Genera mechanism duren by a four-bar linkage.

\begin{tabular}{|c|c|}
\hline$a / h=0.4582$ & $11 \quad 57.98$ \\
\hline$d / b \quad 1.3374$ & $h, h-0.8506$ \\
\hline$\mu_{\mathrm{m} 1 \mathrm{n}}-52 \cdot 18$ & 397 \\
\hline$\overline{B_{0} E_{0}} / b=0.9,316$ & $\mu_{1} \mu_{2}$ \\
\hline$\overline{E_{0} U_{1}} / b-0.4293$ & $2 \tau \quad 90$ \\
\hline
\end{tabular}

for the meaning of the symbols see Fig. 33. 


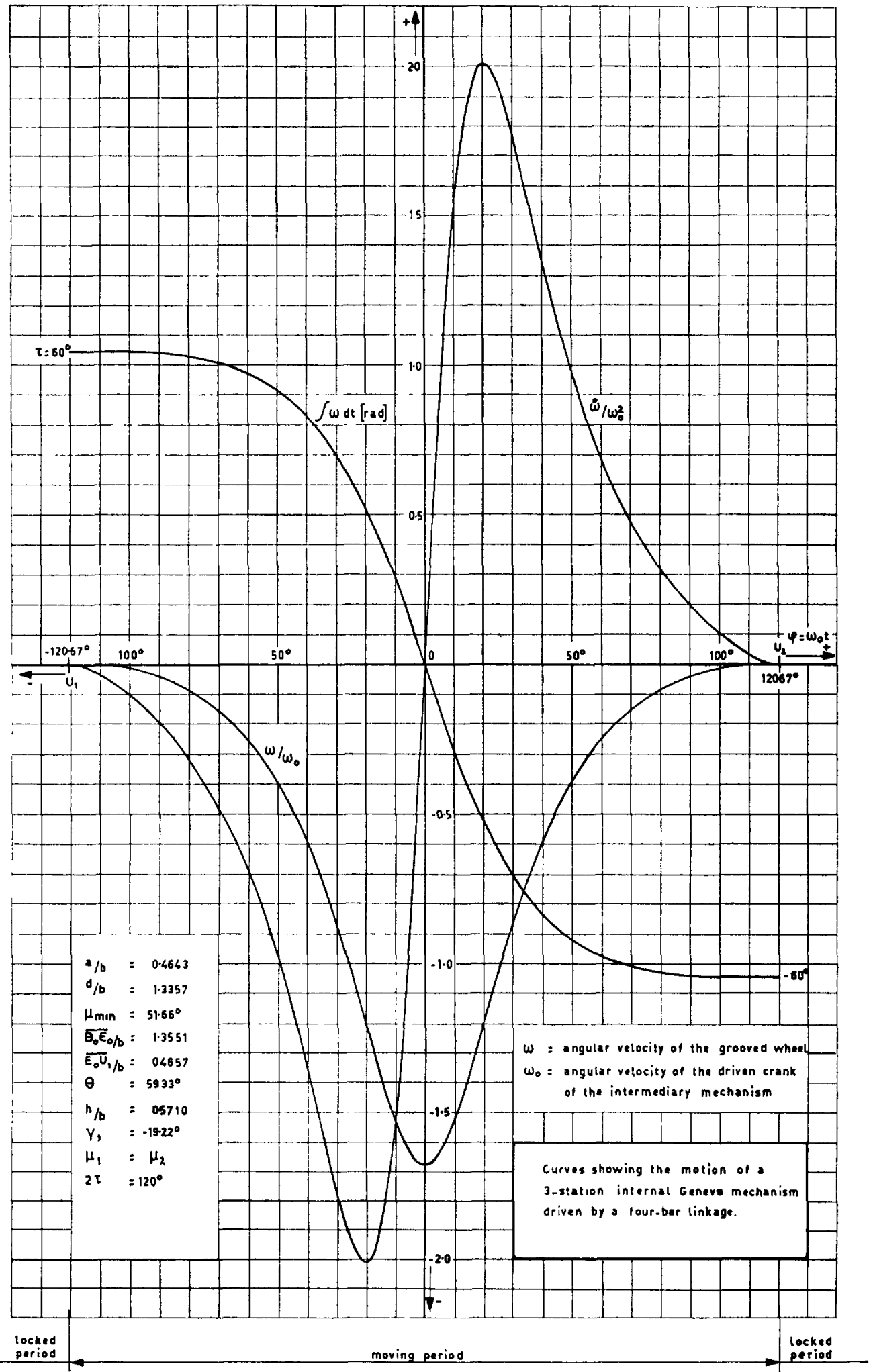

Fio. 43. For the meaning of the symbols see Fig. 33. 

(b) Choose polar co-ordinates $r_{B}$ and $\varphi_{B}$ of the turning-point $B$ with respect to the pole and the pole tangent in such a way that

$$
\frac{1}{2} \sin \varphi_{B}<r_{B} / \delta \leqq \frac{1}{2} \sin \varphi_{B}\left[1+\left(\frac{1-\sin \varphi_{B}}{1+3 \sin \varphi_{B}}\right)^{\frac{1}{2}}\right],
$$

if $B$ is chosen inside the inflection circle, and

$$
r_{B} / \delta \geqq \frac{1}{2} \sin \varphi_{B}\left[1+\left(\frac{1+\sin \varphi_{B}}{1-3 \sin \varphi_{B}}\right)^{\frac{1}{2}}\right],
$$

if $B$ lies outside the inflection circle. (In any case we may confine ourselves to the first quadrant for the choice of $B$.)

(c) Find $B_{0}$ from equation $\overline{P B}^{2}=\overline{B B}_{0} \cdot \overline{B B}_{w}$.

(d) Draw circle $b$ with its centre at $B$ and radius $\overrightarrow{B B}_{0}=b$.

(e) Choose the coupler point in one of the intersections $K_{1}$ and $K_{2}$ of circle $b$ with the inflection circle.

(f) Find the Ball's point $U$ in the chosen coupler point $K$.

(g) Draw a line through $K$ perpendicular to the path normal $P K$. The line segments which this line cuts from the two axes $p$ and $n$ form the sides of a rectangle with $K_{*}$ as the fourth vertex.

(h) Determine point $B_{*}$ in a similar way, starting from point $B$.

(i) Draw line $K_{*} B_{*}$ which intersects $p$ and $n$ at $M$ and $L$ respectively. Then with $\overline{P M}=m$ and $\overline{P L}=l, k_{u}$ is completely determined. (As is shown by Beyer [5], $k_{u}$ is determined by $p, n$ and two points of $k_{u}$, the points being $U$ and $B$ in the case under observation. This is done by determining the line $M L$ as before, from which other points of $k_{u}$ can be derived by reversing the process.)

(j) Point $A$ can then be located at one of the intersection points of $k_{u}$ and circle $b$. As $k_{u}$ is a circular curve, the isotropic points are to be counted as two intersection points. Since we forced Ball's point on $b$ and $k_{u}$, this point of intersection may not be taken as a turning-point. So from the six intersection points of circle $b$ with the cubic $k_{u}$, only three of them remain from which to choose turning-point $A$. Two of them may or may not be complex. If all three intersection points for the choice of $A$ are real and $b$ intersects the inflection circle in two different and real points $K$, six solutions exist in addition to the chosen polar coordinates $r_{B}$ and $\varphi_{B}$ of $B$.

For a real point $A$ on $b$, the added point $A_{*}$ must lie on $K_{*} B_{*}$. Hence this leads to the construction of the real intersection(s) of the cubic $b_{*}$ with $K_{*} B_{*}$, as can be easily effected by way of trial and error.

(k) Find the point $A_{0}$ from point $A$ using the equation $\overline{P A}^{2}=\overline{A A}_{0} \cdot \overline{A A}_{w}$.

(l) Draw the coupler curve, check the position of the axis of symmetry and judge the length of the straight guidance. 
Remarks. The limitation in the choice of the position of turning-point $B$ has three causes:

1. the equivalence of the left- and right-hand half plane;

2. the very existence of real intersections $K_{1}$ and $K_{2}$ of circle $h$ with the inflection circle: and

3. the equivalence of solutions corresponding with the two different positions of the coupler point in the two Ball's points of the coupler curve.

The last point will require some further explanation: The two different inflection circle corresponding to the two positions of the coupler in which the coupler point coincides with a Ball's point, can be made of the same size by increasing or decreasing one of the two fourbar linkages. This makes it possible to indicate the positions of two points $B$ producing equivalent solutions, in one moving plane (of drawing), in which the mutual relation between these points must be investigated.

Let the two positions of the coupler be represented by $A_{1} B_{1}$ and $A_{2} B_{2}$. As is known. the points $U_{1}$ and $U_{2}$ are opposite points to one another with respect to the axis of symmetry of the coupler curve, the latter going through $B_{0}$. The perpendicular bisectors of the distances ${\overline{U_{1}}}_{2}$ and $\bar{B}_{1} B_{2}$ will then intersect at $P_{12}=B_{0}$. So $A_{1}$ and $A_{2}$ are opposite points to one another with respect to the fixed link $A_{0} B_{0}$. To study the relationship between the two positions of the four-bar linkage, it is more convenient to replace the second by its image with respect to the fixed link. The positions under consideration will then be $A_{1} B_{1}$ and $A_{3} B_{3}$, $A_{3}$ coinciding with $A_{1}$. These positions are shown in Fig. 24. It is further proved in part (b) of section 4.3.1 that $P_{1} Q_{1}$ runs parallel to $P_{3} Q_{3}$. From the theorem of Bobillier. it can then be seen that $\Varangle p_{1} P_{1} B_{1}=\Varangle A_{1} P_{1} Q_{1}=\Varangle A_{3} P_{3} Q_{3}=\Varangle p_{3} P_{3} B_{3}$. Hence the two related points $B$, lying in one moving plane as mentioned above, are on the same path normal. It i also proved that ${\overline{P_{1} B_{1}}}_{1} \cdot \bar{P}_{3} B_{3}==\bar{B}_{1} B_{0} \cdot \bar{B}_{3} B_{0}$.

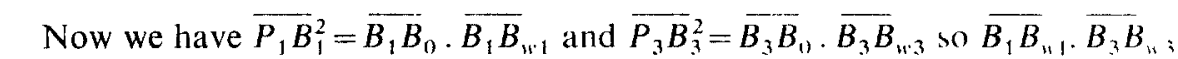
$=\bar{B}_{1} \bar{B}_{0} \cdot \bar{B}_{3} B_{0}$,

or

$$
\left(\delta_{1} \sin \varphi_{B_{1}}-{\overline{P_{1} B_{1}}}_{)}\right)\left(\delta_{3} \sin \varphi_{B_{3}}-{\overline{P_{3} B_{3}}}_{)}={\overline{B_{1} B_{0}}}_{0} \cdot{\overline{B_{3} B_{0}}}_{0}={\overline{P_{1} B_{1}}}_{1}{\overline{P_{3} B_{3}}}_{3}\right.
$$

consequently with $\varphi_{B_{1}}=\varphi_{B_{2}}$, for the two four-bar linkages the following relation hold:

$$
\frac{\bar{P}_{1} B_{1}}{\delta_{1}}-\frac{1}{2} \sin \varphi_{B 1}=2 \sin \varphi_{B_{1}}-\frac{\left(\bar{P}_{3} B_{3} \delta_{1} / \delta_{3}\right)}{\delta_{1}}
$$

In the moving plane of drawing the two couplers have the same inflection circle: so for the two related points $B$ in this moving plane we have the equation

$$
r_{B}-\frac{1}{2} \delta \sin \varphi_{B}=\frac{1}{2} \delta \sin \varphi_{B}-r_{B} .
$$

Hence, the midpoint between two related points $B$ of the moving plane must coincide with the intersection of a path normal with a circle which touches the pole tangent in $P$ and passes through the centre of the inflection circle (see Fig. 45). (In general this midpoint does not coincide with $P$.) The equations referring to this general case have been omitted. They can be found in the author's thesis [2]. 


\section{REFERENCES}

[1] K. H. HUNT, N. FinK and J. NAYAR, Linkage Geneva mechanisms. A design study in mechanism geometry. Proc. Mech. Inst. Engrs. 174, 643 (1960).

[2] E. A. Duxsman, The Four-Bar Linkage as a Driving Mechanism (in Dutch). The Technical Publishers, H. Stam (1964).

[3] A. S. HaLL, Kinematics and Linkage Design, pp. 97-105. Prentice-Hall (1961).

[4] Sir Robert Ball, Dublin Proc. (2) 1, 243 (1871).

[5] R. BEYER, The Kinematic Synthesis of Mechanisms (translated from the German by H. Kuenzel), p. 208. Chapman \& Hall (1963).

[6] J. HirschHORN Kinematics and Dynamics of Plane Mechanisms, pp. 349-356. McGraw-Hill (1962).

[7] K. Rauh and L. HagedoRn, Praktische Getriebelehre I: Die Viergelenkkette (3rd edition), Vol. 1, pp. 95-96. Springer-Verlag (1965). 